\title{
Coordinated post-contingency dispatch of integrated energy system with multiple participants based on distributed energy trading
}

\author{
Wang, Can; Bie, Zhaohong; Wu, Qiuwei; Li, Gengfeng; Xie, Haipeng
}

Published in:

International Journal of Electrical Power and Energy Systems

Link to article, DOI:

10.1016/j.jjepes.2021.107011

Publication date:

2021

Document Version

Early version, also known as pre-print

Link back to DTU Orbit

Citation (APA):

Wang, C., Bie, Z., Wu, Q., Li, G., \& Xie, H. (2021). Coordinated post-contingency dispatch of integrated energy system with multiple participants based on distributed energy trading. International Journal of Electrical Power and Energy Systems, 130, [107011]. https://doi.org/10.1016/j.ijepes.2021.107011

\section{General rights}

Copyright and moral rights for the publications made accessible in the public portal are retained by the authors and/or other copyright owners and it is a condition of accessing publications that users recognise and abide by the legal requirements associated with these rights.

- Users may download and print one copy of any publication from the public portal for the purpose of private study or research.

- You may not further distribute the material or use it for any profit-making activity or commercial gain

- You may freely distribute the URL identifying the publication in the public portal 


\title{
Coordinated post-contingency dispatch of integrated energy system with multiple participants based on distributed energy trading
}

\author{
Can Wang ${ }^{\mathrm{a}}$, Zhaohong Bie ${ }^{\mathrm{a}, *}$, Qiuwei Wu ${ }^{\mathrm{b}}$, Gengfeng $\mathrm{Li}^{\mathrm{a}}$, Haipeng Xie \\ ${ }^{a}$ State Key Laboratory of Electrical Insulation and Power Equipment, Smart Grid Key Laboratory of Shaanxi Province, Department of \\ Electrical Engineering, Xi'an Jiaotong University, Xi'an, 710049, China \\ ${ }^{b}$ Centre for Electric Power and Energy, Department of Electrical Engineering, Technical University of Denmark, Kgs. Lyngby, Denmark 2800
}

\begin{abstract}
The electrical, natural gas and heating subsystems of an integrated energy system (IES) can be managed by multiple independent energy operators (participants), individually. In the face of contingency events, it is essential to coordinate these participants, who pursue their own profits, in order to optimize the post-contingency dispatch of the IES and reduce the system loss. Therefore, this paper proposes a novel coordinated post-contingency dispatch scheme for the IES with multiple participants. Firstly, an emergency energy market framework of the IES is developed based on a distributed energy trading mechanism, to coordinate the energy allocation among different participants during contingencies. Then, the post-contingency dispatch model of the IES is formulated to maximize the total social welfare of the IES during contingencies. Moreover, a distributed optimization algorithm for the IES is developed based on the alternating direction method of multipliers (ADMM) with self-adaptive penalty parameters, which provides the optimal emergency energy prices to effectively coordinate the decision-making process of participants. Finally, through case studies, the proposed method is compared to the traditional centralized dispatch method and decentralized method based on regulated energy prices to demonstrate the effectiveness of the proposed method.
\end{abstract}

Keywords: Integrated energy system; Post-contingency dispatch; Load shedding; Energy market; Distributed optimization.

\begin{tabular}{|llll|}
\hline \multicolumn{2}{|l}{ Nomenclature } & & \\
A. Abbreviations & $s_{i j, t}, s_{\varphi, t}^{S h}$ & State of branch $i j$ and compensator $\varphi$ at $t$ \\
IES & Integrated energy system & $s_{z, t}^{G S}, s_{m n, t}$ & State of gas source $z$ and pipeline $m n$ at $t$ \\
ADMM & Alternating direction method of multipliers & $s_{\kappa, t}^{G C}$ & State of gas compressor $\kappa$ at time $t$ \\
ESO & Electricity system operator & $s_{a b, t}^{s p}, s_{a b, t}^{r t}$ & State of supply/return pipeline $a b$ at time $t$ \\
GSO & Gas system operator & $s_{c, t}^{C F B}, s_{w, t}^{E B}$ & State of coal-fired boiler $c$ and EB $w$ at $t$ \\
HSO & Heating system operator & $s_{v, t}^{G E}, s_{d, t}^{G B}$ & State of GE $v$ and GB $d$ at time $t$ \\
EETP & Emergency energy trading platform & $t_{s t a r t}, t_{e n d}$ & The occurrence and predicted end time of a \\
EB & Electric boiler & $C^{g r i d}$ & Electricity price of the upper grid \\
GE & Gas-fired generator & $C_{o}^{E S S}$ & The depreciation cost of battery $o$ \\
GB & Gas boiler & $C_{y}^{R E}$ & The unit operation cost of renewable energy $y$ \\
\hline
\end{tabular}

* Corresponding author. E-mail address: zhbie@mail.xjtu.edu.cn 


\begin{tabular}{|c|c|c|c|}
\hline \multicolumn{2}{|c|}{ B. Indices and sets } & \multirow{2}{*}{$\begin{array}{l}C_{z}^{G S} \\
C_{c}^{C F B}\end{array}$} & \multirow{2}{*}{$\begin{array}{l}\text { Natural gas price of the gas source } z \\
\text { The unit operation cost of coal-fired boiler } c\end{array}$} \\
\hline$t$ & Time interval (hours) & & \\
\hline$i, j, \Omega^{E l e}$ & Index and set of electric buses & $L_{e, j, t}^{P}, L_{e, j, t}^{Q}$ & Active/reactive load on the bus $j$ at time $t$ \\
\hline$m, n, \Omega^{\text {Gas }}$ & Index and set of nodes in gas networks & $L_{g, m, t}$ & Gas load on the node $m$ at time $t$ \\
\hline$a, b, \Omega^{\text {Heat }}$ & Index and set of nodes in heating networks & $L_{h, a, t}$ & Heat load on the node $a$ at time $t$ \\
\hline$\Omega^{E S S}, \Omega^{R E}$ & Set of batteries and renewable energy & $r_{i j}, x_{i j}$ & Resistance and reactance of electric branch ij \\
\hline$\Omega^{S h}, \Omega^{b r}$ & Set of compensators and electric branches & $\eta_{c h, o}^{E S S}, \eta_{d c h, o}^{E S S}$ & Charging/discharging efficiency of battery $o$ \\
\hline$\Omega^{G P}, \Omega^{G C}$ & Set of gas pipelines and compressors & $\eta_{v}^{G E}$ & Conversion efficiency coefficient of GE $v$ \\
\hline$\Omega^{G S}, \Omega^{C F B}$ & Set of gas sources and coal-fired boilers & $\eta_{w}^{E B}$ & The heating efficiency of EB $w$ \\
\hline$\Omega_{H P}^{s p}, \Omega_{H P}^{r t}$ & Set of supply and return heating pipelines & $\eta_{d}^{G B}$ & The heating efficiency of GB $d$ \\
\hline$\Omega^{E B}$ & Set of electric boilers & $\eta_{m n}$ & Weymouth constant of gas pipeline $m n$ \\
\hline$\Omega^{G E}$ & Set of gas-fired generators & $\eta_{\kappa}^{G C}$ & Gas consumption coefficient of compressor $\kappa$ \\
\hline$\Omega^{G B}$ & Set of gas boilers & $\Phi_{\kappa}^{\max }$ & Maximal compression ratio of compressor $\kappa$ \\
\hline$f(j), t(j)$ & Set of parents and children buses of bus $j$ & $\eta_{a b}^{s p}$ & esistance coefficient of supply pipeline $a b$ \\
\hline$f(a), t(a)$ & Set of parents and children nodes of node $a$ & $\eta_{a b}^{r t}$ & Resistance coefficient of return pipeline $a b$ \\
\hline C. Variables & & $T_{a, t}^{s p}, T_{a, t}^{r t}$ & Supply/return sides temperatures of node $a$ \\
\hline$P_{t}^{\text {grid }}, Q_{t}^{\text {grid }}$ & Active/reactive power of upper grid at time $t$ & $P^{g r i d, \max / \min }$ & Maximal/minimal active power of upper grid \\
\hline$P_{c h, o, t}^{E S S}, P_{d c h, o, t}^{E S S}$ & Charging/discharging power of battery $o$ at $t$ & $Q^{g r i d, \max / \min }$ & Maximal/minimal reactive power of upper grid \\
\hline$P_{y, t}^{R E}$ & Power of renewable generation $y$ at time $t$ & $P_{y}^{R E, \min }$ & Minimal active power of renewable energy $y$ \\
\hline$P_{i j, t}, Q_{i j, t}$ & Active/reactive power flow in branch $i j$ at $t$ & $P_{y, t}^{R E, p r e}$ & $\begin{array}{l}\text { le active power of renewable } \\
t\end{array}$ \\
\hline$l_{i j, t}$ & Line current square of branch $i j$ at time $t$ & $P_{c h, o}^{E S S, \max }, P_{d c h, o}^{E S S, \max }$ & Maximal charging/ discharging power \\
\hline$Q_{\varphi, t}^{S h}$ & Reactive power of compensator $\varphi$ at time $t$ & $E_{o}^{E S S, \max / \min }$ & Maximal/minimal stored energy in battery $o$ \\
\hline$L_{e, j, t}^{c r t, P}, L_{e, j, t}^{c r t, Q}$ & Active/reactive load shedding on bus $j$ at $t$ & $Q_{\varphi}^{S h, \text { max }}, Q_{\varphi}^{S h, \min }$ & Maximal/minimal reactive power of $\varphi$ \\
\hline$E_{o, t}^{E S S}$ & Stored energy in battery $o$ at time $t$ & $l_{i j}^{\max }$ & square of branch $i j$ \\
\hline$u_{i, t}$ & Voltage magnitude square on bus $i$ at time $t$ & $u_{j}^{\max }, u_{j}^{\min }$ & Maximal/minimal voltage magnitude square \\
\hline$R_{i j, t}, \xi^{\sigma}, \psi^{\sigma}$ & $\begin{array}{l}\text { Introduced variables for the polyhedral } \\
\text { approximation }\end{array}$ & $G_{z}^{G S, \max }, G_{z}^{G S, \min }$ & Maximal/minimal gas supply of gas source $z$ \\
\hline$G_{z, t}^{G S}$ & Gas supply of gas source $z$ at time $t$ & $G_{m n}^{\max }$ & Maximal gas flow in gas pipeline $m n$ \\
\hline$G_{m n, t}$ & Gas flow in pipeline $m n$ at time $t$ & $G_{\kappa}^{G C}$ & $\begin{array}{l}\text { Maximal/minimal transportation capacity of } \\
\text { compressor } \kappa\end{array}$ \\
\hline$G_{s u c, \kappa, t}^{G C}, G_{d i s, \kappa, t}^{G C}$ & Suction/discharge gas flow of compressor $\kappa$ & $\Pi_{m}^{\max }, \Pi_{m}^{\min }$ & Maximal/minimal gas pressure of node $m$ \\
\hline$\Pi_{\text {suc }, \kappa, t}^{G C}, \Pi_{d i s, \kappa, t}^{G C}$ & $\begin{array}{l}\text { Pressure at the inlet and outlet nodes of gas } \\
\text { compressor } \kappa \text { at time } t\end{array}$ & $H_{c}^{C F B, \max / \min }$ & Maximal/minimal heat power of boiler $c$ \\
\hline$\Pi_{m, t}, \Pi_{n, t}$ & Gas pressure of node $m$ and $n$ at time $t$ & $M_{a b}^{s p, \max }, M_{a b}^{r t, \max }$ & Maximal mass flow of supply/return pipeline \\
\hline$L_{g, m, t}^{c r t}$ & Gas load curtailment on node $m$ at time $t$ & $\Gamma_{a}^{s p, \max / \min }$ & $\begin{array}{l}\text { Maximal } / \text { minimal pressures at the supply side } \\
\text { of node } a\end{array}$ \\
\hline$H_{c, t}^{C F B}$ & Heat power of coal-fired boiler $c$ at time $t$ & $\Gamma_{a}^{r t, \max / \min }$ & $\begin{array}{l}\text { Maximal } / \text { minimal pressures at the return side } \\
\text { of node } a\end{array}$ \\
\hline$H_{i n j, a, t}$ & $\begin{array}{l}\text { Injected heat power of node } a \text { from other } \\
\text { nodes at time } t\end{array}$ & $P_{v}^{G E, \max }, P_{v}^{G E, \min }$ & Maximal/minimal electric power of GE $v$ \\
\hline$M_{a b, t}^{s p}, M_{a b, t}^{r t}$ & Mass flow rate in supply/return pipeline $a b$ & $G_{v}^{G E, \max }, G_{v}^{G E, \min }$ & Maximal/minimal gas demand of GE $v$ \\
\hline$\Gamma_{a, t}^{s p}, \Gamma_{a, t}^{r t}$ & Pressures in the supply/return side of node $a$ & $H_{w}^{E B, \max }, H_{w}^{E B, \min }$ & Maximal/minimal heat power of EB $w$ \\
\hline
\end{tabular}




\begin{tabular}{|c|c|c|c|}
\hline$L_{h, a, t}^{c r t}$ & Heat load curtailment on node $a$ at time $t$ & $P_{w}^{E B, \max }, P_{w}^{E B, \min }$ & Maximal/minimal electric demand of $\mathrm{EB} w$ \\
\hline$P_{v, t}^{G E}, G_{v, t}^{G E}$ & Electric power and gas demand of GE $v$ at $t$ & $H_{d}^{G B, \max }, H_{d}^{G B, \min }$ & Maximal/minimal heat power of GB $d$ \\
\hline$H_{w, t}^{E B}, P_{w, t}^{E B}$ & Heat power and electric demand of EB $w$ at $t$ & $G_{d}^{G B, \max }, G_{d}^{G B, \min }$ & Maximal/minimal gas demand of GB $d$ \\
\hline$H_{d, t}^{G B}, G_{d, t}^{G B}$ & Heat power and gas demand of GB $d$ at $t$ & $\varsigma$ & Special heat capacity of water \\
\hline \multicolumn{2}{|c|}{ D. Parameters } & $B$ & A large positive constant \\
\hline$S_{y, t}^{R E}, S_{o, t}^{E S S}$ & State of renewable energy and batteries at $t$ & $G H V$ & Natural gas gross heating value \\
\hline
\end{tabular}

\section{Introduction}

Recently, to promote the utilization of renewable energy and enhance the reliability and flexibility of energy systems, the concept of the integrated energy system (IES) is proposed as a promising future urban or regional energy system [1], [2]. In the IES, previously independent electrical, heating, and natural gas networks are interlinked by various energy conversion components, e.g., gas-fired generators (GE), electric boilers (EB) and gas boilers (GB). To unlock the benefits of energy network integration, the optimal dispatch of the IES is extremely important. Especially, when the IES encounters contingency events (e.g., the failures of important components) that can lead to severe energy shortage, the post-contingency dispatch of the IES is essential, which can fully dispatch various energy resources to supply important loads, thereby minimizing the economic loss of the IES during contingencies.

In the existing literature, there have been several studies on the optimal dispatch of the IES [3-7]. In [3], a day-ahead economic dispatch model of IES with reserve scheduling was presented considering the uncertainties from renewable energy. In [4], a stochastic day-ahead scheduling model of the IES was proposed, which develops a method to identify redundant gas network constraints. Moreover, Yu et al. [5] presented an optimization model to minimize the electricity, heat, and gas load shedding of the IES during component failures. In [6], an optimal load shedding model of the IES was presented considering power-to-gas devices and gas storages. Guo et al. [7] proposed a resilience-oriented stochastic configuration framework for the IES considering the integrated demand response, thereby reducing the worst-case cost in case of emergency events.

The aforementioned studies mainly focus on the post-contingency dispatch of the IES in a centralized manner, which needs a centralized operator to coordinate and dispatch various resources of the entire system. Recently, to reduce management complexity and protect information privacy, the decentralized operation mode of the IES has been widely adopted, where the electrical, gas and heating subsystems of an IES can be managed by multiple energy operators individually [8], [9]. Each energy operator is an independent participant of the IES. Due to the independence of energy operators, each participant tries to maximize its own profits and cannot directly dispatch other participants. Therefore, when the IES encounters contingencies or emergency situations, each participant intends to obtain an adequate energy supply to minimize its load shedding. Nevertheless, due to the impacts of component failures, the energy supply of IES 
may be very limited during contingencies, which will cause conflicts among the dispatch strategies of different participants [10]. Hence it is necessary to develop an effective post-contingency dispatch scheme to coordinate different participants and preferentially allocate the limited energy resources to the most important loads, thereby maximizing the social welfare of the entire IES during contingencies. However, the related research is rather limited so far.

In the previous studies on electricity systems, the market mechanism provides an effective method to coordinate the energy dispatch of different entities [11]-[14]. Kim et al. [11] presented a framework of direct energy trading for multiple microgrids to stimulate the energy share among microgrids, thereby maximizing social welfare. In [12], a peer-to-peer energy market of distribution networks was formulated based on the multi-class energy management, and the alternating direction method of multipliers (ADMM) was employed to coordinate the trading between prosumers and the wholesale electricity market. In [13], a multi-agent transactive energy management framework for distribution systems was proposed, which motivates the participation of demand response. Besides, an emergency market mechanism for the multi-microgrid systems was presented in [14], which coordinates the electricity dispatch among microgrids during contingency events.

As an emerging attractive concept, the multi-energy market of the IES has attracted considerable attention, which has a promising potential in optimizing the energy management of the IES [15]-[18]. Sorknæs et al. [15] introduced the concept of Smart Energy Markets, which integrates the electricity, heating, gas and fuel markets in future energy systems. Stiphout et al. [16] quantitatively analyzed the benefits of a multi-energy market by taking the advantages of energy system integration. In [17], a cooperative optimal operation model for multiple energy hubs was developed based on game theory. Furthermore, in [18], a bilateral gas-electricity market was presented based on locational marginal prices, which enhances the operational flexibility of gas and electricity systems.

The above studies provide valuable references for the optimal dispatch of the IES. However, they mainly focus on the operation of IES under normal conditions. During contingencies, the network topology of the IES may be changed and the energy supply capacity of the IES may decrease dramatically [19], which will lead to inevitable load shedding to ensure system security. Thus, in the post-contingency dispatch, the financial aspects of load shedding have considerable impacts on the decision-making of participants, which is generally neglected in the previous energy market framework of the IES. Moreover, due to the impacts of failures, several common assumptions of the IES under normal conditions are no longer valid during contingencies, e.g., the constant-flow mode of heating networks [20]. Overall, to the best of the authors' knowledge, the coordinated post-contingency dispatch for the IES with multiple participants has not been studied so far.

Therefore, this paper proposes a novel coordinated post-contingency dispatch scheme for multiple participants of the 
IES to reduce the economic loss of the entire IES in face of contingencies. The multi-energy markets are integrated with the emergency energy dispatch of the IES to effectively stimulate the participants to share their resources with other participants during contingencies. Besides, considering the impacts of contingencies, the economic cost of load shedding is treated as an important concern in the energy dispatch of participants, and the mathematical models of electricity, gas and heating networks during contingencies are formulated. Furthermore, to coordinate the energy allocation among participants, a distributed emergency energy trading algorithm for the IES is developed. The main contributions are summarized as below:

1) A novel emergency energy market framework is proposed for the IES, which coordinates the energy dispatch among the electricity system operator (ESO), gas system operator (GSO) and heating system operator (HSO) during contingency events.

2) The post-contingency dispatch scheme of the IES is developed considering the energy network models and impacts of various component failures, which aims at minimizing the total operation cost of the IES during contingencies. Several linearization techniques are used to reduce the computational complexity of non-convex energy network models.

3) A distributed emergency energy trading algorithm for the IES is developed based on the ADMM with selfadaptive penalty parameters, which can provide the emergency energy trading prices to coordinate the energy allocation among different participants, as well as ensuring the privacy and profits of each participant.

The rest of this paper is organized as follows. Section 2 presents the emergency energy market framework for IES. In Section 3, the post-contingency dispatch model of IES is proposed. Section 4 describes the distributed emergency energy trading algorithm. Section 5 verifies the effectiveness of the proposed method via extensive case studies. Finally, Section 6 concludes this paper.

\section{The emergency energy market framework for IES}

This paper presents a novel emergency energy market framework to coordinate the energy dispatch among different participants of IES during contingencies. To simplify the illustration, we choose a typical mode of IES which contains an electricity system, a natural gas system and a heating system as the example [21]. The electricity system, gas system and heating system of IES are managed by one ESO, GSO and HSO, respectively. The diagram of the IES is shown in Fig. 1.

As shown in Fig. 1, in the IES, the ESO purchases electricity from the upper grid and dispatches the renewable energy and batteries to supply the load of electric consumers. If the electricity supply is insufficient, ESO can also buy natural gas from GSO to run gas-fired generators. GSO supplies the load of natural gas consumers using the output of gas sources, as well as providing gas supply to ESO and HSO. And HSO purchases gas from GSO to run gas boilers and 
buys electricity from ESO to run electric boilers, thereby supplying the load of heat consumers.

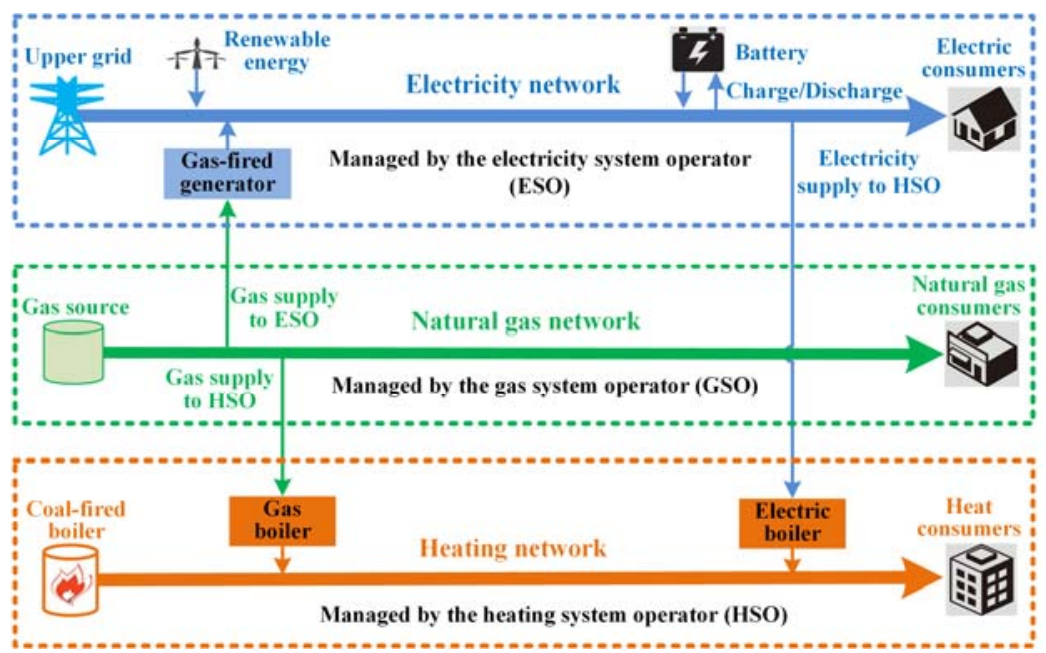

Fig. 1 The diagram of an IES with multiple participants

During contingency events (e.g., the outage of the upper grid), the IES may face severe energy shortage. And according to the studies on the reliability of IES [6, 22], the failures of components generally can last for several hours. It means the post-contingency dispatch problem of IES is an optimal operation problem in the duration of contingency events. In post-contingency dispatch, the limited energy resources should be preferentially allocated to the most important loads, thereby maximizing total social welfare [14]. However, since these participants are independent, ESO may be unwilling to curtail the non-critical load of electric consumers to preferentially supply the electric demand of $\mathrm{HSO}$, if ESO is not fairly compensated. It will hinder the emergency energy allocation of the entire IES. To coordinate the emergency energy allocation among these participants, an emergency energy market framework of IES is developed based on a distributed energy trading mechanism. The block diagram of the proposed market for IES is shown in Fig. 2.

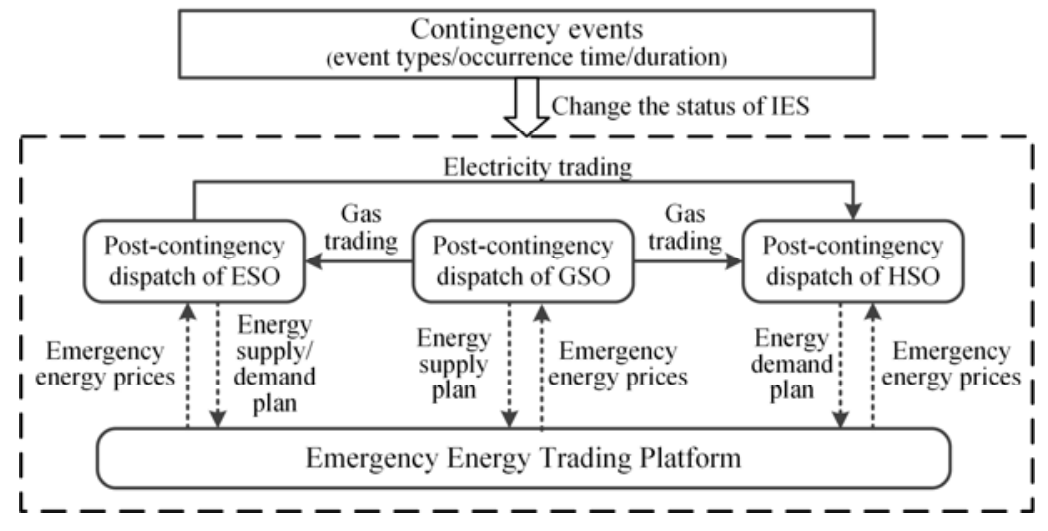

Fig. 2 The diagram of the emergency energy market for the IES

In the face of contingency events, the ESO, GSO and HSO will respectively make post-contingency dispatch decisions according to the event types, occurrence time and predicted duration of contingencies, thereby minimizing their 
operation cost (including the economic cost of load shedding). Besides, in post-contingency dispatch, one participant can sell/purchase energy to/from other participants, to fully utilize its surplus energy or supplement the energy shortage. As previously mentioned, due to the independence of participants, there may be a mismatch between the energy demand of buyers and the supply of sellers during contingencies, which will hinder the emergency energy trading of IES. Therefore, to coordinate the distributed energy trading among these participants, an emergency energy trading platform (EETP) is introduced in this paper. This platform acts as an auctioneer [12] (not a control center) in the emergency energy market of IES, which can adjust the emergency energy prices to balance the energy demand of buyers and the supply of sellers. Based on the emergency market framework, the corresponding energy trading and settlement process is shown in Fig. 3.

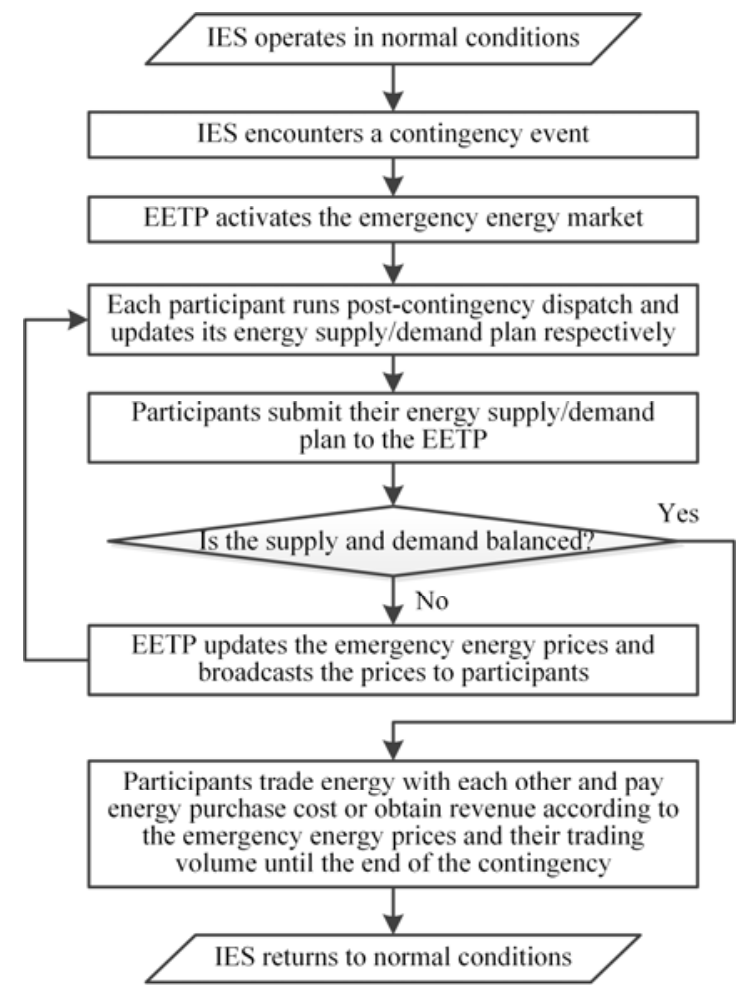

Fig. 3 The energy trading and settlement process of the emergency energy market for IES

As shown in Fig. 3, when the IES encounters a contingency event, the emergency energy market will be activated. Then, each participant conducts the post-contingency dispatch respectively. Afterward, participants need to submit their energy supply/demand plan (in the duration of contingency) to the EETP. If the demand of buyers and the supply of sellers are unbalanced, the EETP will adjust the emergency energy prices at energy coupling components and broadcast the updated energy prices to participants, thereby stimulating participants to regulate their demand/supply plan individually. After several iterations, the demand plan of buyers and the supply plan of sellers balance, and the emergency energy prices stop changing. Then participants will trade energy with each other and pay corresponding energy purchase cost or obtain revenue according to the last updated energy prices until the end of the contingency. 
Finally, the emergency energy market is shut down and the IES returns to normal conditions. Overall, the designed emergency energy market of IES has the following features:

1) Optimize the post-contingency dispatch of IES: In the designed emergency energy market, the participant who needs to supply the most important loads is willing to accept the highest emergency energy price, thereby purchasing more energy from other participants. It will result in the limited energy resources of IES are preferentially allocated to the most important loads, which can maximize the social welfare of the entire IES during contingencies.

2) Stimulate participants to share energy voluntarily with economic measures: In post-contingency dispatch, the EETP stimulates the coordination of participants by adjusting the emergency energy prices. To obtain more revenue, one participant will voluntarily curtail its less important loads to supply the more important demand of other participants, without requiring mandatory intervention.

3) Protect the information privacy of each participant: In the designed market framework, the participants of IES make post-contingency dispatch decisions independently. Therefore, only limited information needs to be exchanged among participants and the EETP. A vast amount of private information data of participants (e.g., the data of consumers) will not be shared among participants. It can also reduce the communication burden and the delay of communication.

In this section, we introduce the framework of the designed emergency energy market for the IES. However, it is noteworthy that the proposed market framework is not limited to the IES with only one ESO, GSO and HSO. Based on the structure of distributed energy trading among participants, the proposed emergency energy market is also applicable to the IES with multiple electrical subsystems, gas subsystems and heating subsystems.

\section{The post-contingency dispatch model of the IES}

In this section, the mathematical model for the post-contingency dispatch of IES is presented, which aims to maximize the total social welfare of IES during contingency events. It is worth noting that the post-contingency dispatch model of IES is presented in a centralized formulation in this section. And in Section 4, the decentralized solution algorithm for this model based on the proposed emergency market framework will be introduced. Moreover, in the postcontingency dispatch, it is necessary to conduct the optimal energy dispatch decisions as rapidly as possible, thereby effectively reducing the loss caused by contingency events. However, the mathematical models of electricity, gas, and heating networks generally are non-convex and nonlinear, which brings huge computational complexity. Therefore, to speed up the calculation of the post-contingency dispatch model, several linearization techniques are used to reduce the computational complexity of non-convex energy network models.

\subsection{Objective Function}

The objective function of the post-contingency dispatch model for IES is to minimize the total operation cost of IES 
in the duration of contingencies, which means maximizing the total social welfare. The total operation cost in (1) contains the electricity generation cost, the depreciation cost of batteries [23], the supply cost of gas sources, the operation cost of coal-fired boilers, as well as the economic cost of electric, gas and heat load shedding.

$$
\begin{aligned}
\min \sum_{t=t_{\text {sort }}}^{t_{\text {tond }}}\left(C^{g r i d} P_{t}^{g r i d}+\sum_{y \in \Omega^{R E}} C_{y}^{R E} P_{y, t}^{R E}+\sum_{o \in \Omega^{E S S}} C_{o}^{E S S}\left(P_{c h, o, t}^{E S S}+P_{d c h, o, t}^{E S S}\right)+\sum_{z \in \Omega^{C S}} C_{z}^{G S} G_{z, t}^{G S}+\sum_{c \in \Omega^{C E B}} C_{c}^{C F B} H_{c, t}^{C F B}\right. \\
\left.+\sum_{j \in \Omega^{E l e}} C_{e, j}^{c r t} L_{e, j, t}^{c r t, P}+\sum_{m \in \Omega^{c o s}} C_{g, m}^{c r t} L_{g, m, t}^{c r t}+\sum_{a \in \Omega^{H e a t}} C_{h, a}^{c r t} L_{h, a, t}^{c r t}\right)
\end{aligned}
$$

where $C_{e, j}^{c r t}$ denotes the curtailment cost of the electric load on bus $j, C_{g, m}^{c r t}$ is the curtailment cost of gas load on node $m$, and $C_{h, a}^{c r t}$ is the curtailment cost of heat load on node $a$. In post-contingency dispatch, the more important the load of a consumer is, the higher its load curtailment cost is, which can result in that the limited energy resources will be preferentially allocated to the most important loads $[10,24]$.

\subsection{The electricity system constraints}

Generally, the electricity system of an IES is the distribution system. Therefore, the AC power flow model [25] is employed in this paper. The active power balance (2) and reactive power balance (3) are as follows:

$$
\begin{gathered}
\varpi_{j} P_{t}^{\text {grid }}+\sum_{y \in \Omega_{j} \in} P_{y, t}^{R E}+\sum_{o \in \Omega_{j}^{E S S}}\left(P_{d c h, o, t}^{E S S}-P_{c h, o, t}^{E S S}\right)-\sum_{i \in t(j)} P_{j i, t}+\sum_{i \in f(j)}\left(P_{i j, t}-r_{i j} l_{i j, t}\right)+\sum_{v \in \Omega_{j}^{G E}} P_{v, t}^{G E}-\sum_{w \in \Omega_{j}^{E B}} P_{w, t}^{E B}=L_{e, j, t}^{P}-L_{e, j, t}^{c r t, P}, \forall t, \forall j \in \Omega^{E l e}(2) \\
\varpi_{j} Q_{t}^{g r i d}+\sum_{\varphi \in \Omega_{j}^{S h}} Q_{\varphi, t}^{S h}-\sum_{i \in t(j)} Q_{j i, t}+\sum_{i \in f(j)}\left(Q_{i j, t}-x_{i j} l_{i j, t}\right)=L_{e, j, t}^{Q}-L_{e, j, t}^{c r t, Q}, \quad \forall t, \forall j \in \Omega^{E l e}
\end{gathered}
$$

If the electric bus $j$ is connected to the upper grid, the parameter $\varpi_{j}$ equals to 1 ; otherwise, it equals to 0 . $\Omega_{j}^{R E}, \Omega_{j}^{E S S}, \Omega_{j}^{G E}$ and $\Omega_{j}^{E B}$ denote the set of renewable energy generators, batteries, gas-fired generators and electric boilers connected to the bus $j$, respectively.

During contingency events, the components of electricity system may be in the outage state, which has severe impacts on system operation. Considering the impacts of component failures, the parameters which denote the states of components are introduced to the system constraints. For instance, in (4), $s_{t}^{\text {grid }}$ is a parameter which denotes the state of the upper grid at time $t$. If the upper grid is in the normal condition, $s_{t}^{\text {grid }}=1$ and the active power of upper grid will be limited between its maximal and minimal values; if the upper grid is in the outage state, $s_{t}^{\text {grid }}=0$ and the power of upper grid will be limited to 0 . Besides, in (16)-(19), the parameter $s_{i j, t}$ denotes the state of the electric branch $i j$ at time $t$. If the branch $i j$ is in the outage state, $s_{i j, t}=0$ and the active and reactive power flow in the branch $i j$ will be limited to 0 according to (17). Moreover, since the parameter $B$ is a large positive constant and $s_{i j, t}$ equals 0 , the constraint (19) which refers to the relationship between the power flow in branches and the voltage of their connected buses will be 
relaxed for the branch $i j$. Similarly, the states of other components are considered in system constraints, as follows:

$$
\begin{aligned}
& s_{t}^{\text {grid }} P^{\text {grid,min }} \leq P_{t}^{\text {grid }} \leq s_{t}^{\text {grid }} P^{\text {grid,max }}, \quad \forall t \\
& S_{t}^{\text {grid }} Q^{\text {grid,min }} \leq Q_{t}^{\text {grid }} \leq s_{t}^{\text {grid }} Q^{\text {grid,max }}, \quad \forall t \\
& s_{y, t}^{R E} P_{y}^{R E, \text { min }} \leq P_{y, t}^{R E} \leq s_{y, t}^{R E} P_{y, t}^{R E, p r e}, \quad \forall t, \forall y \in \Omega^{R E} \\
& 0 \leq P_{c h, o, t}^{E S S} \leq s_{o, t}^{E S S} P_{c h, o}^{E S S, \max }, \quad \forall t, \forall o \in \Omega^{E S S} \\
& 0 \leq P_{d c h, o, t}^{E S S} \leq S_{o, t}^{E S S} P_{d c h, o}^{E S S, \max }, \quad \forall t, \forall o \in \Omega^{E S S} \\
& E_{o, t+1}^{E S S}=E_{o, t}^{E S S}+\left(\eta_{c h, o}^{E S S} P_{c h, o, t}^{E S S}-\frac{1}{\eta_{d c h, o}^{E S S}} P_{d c h, o, t}^{E S S}\right), \forall t, \forall o \in \Omega^{E S S} \\
& E_{o}^{E S S, \text { min }} \leq E_{o, t}^{E S S} \leq E_{o}^{E S S, \max }, \quad \forall t, \forall o \in \Omega^{E S S} \\
& E_{o, t_{\text {start }}}^{E S S}-E_{o, t_{\text {end }}}^{E S S}=0, \quad \forall o \in \Omega^{E S S} \\
& s_{v, t}^{G E} P_{v}^{G E, \min } \leq P_{v, t}^{G E} \leq s_{v, t}^{G E} P_{v}^{G E, \text { max }}, \quad \forall t, \forall v \in \Omega^{G E} \\
& P_{v, t}^{G E}=\eta_{v}^{G E} G_{v, t}^{G E} \times G H V, \quad \forall t, \forall v \in \Omega^{G E} \\
& P_{w}^{E B, \text { min }} \leq P_{w, t}^{E B} \leq P_{w}^{E B, \text { max }}, \quad \forall t, \forall w \in \Omega^{E B} \\
& s_{\varphi, t}^{S h} Q_{\varphi}^{S h, \text { min }} \leq Q_{\varphi, t}^{S h} \leq s_{\varphi, t}^{S h} Q_{\varphi}^{S h, \text { max }}, \quad \forall t, \forall \varphi \in \Omega^{S h} \\
& P_{i j, t}^{2}+Q_{i j, t}^{2}=u_{i, t} l_{i j, t}, \quad \forall t, \forall i j \in \Omega^{b r}, \text { if } s_{i j, t}=1 \\
& P_{i j, t}=0, \quad Q_{i j, t}=0, \quad \forall t, \forall i j \in \Omega^{b r}, \text { if } s_{i j, t}=0 \\
& 0 \leq l_{i j, t} \leq s_{i j, t} l_{i j}^{\max }, \quad \forall t, \forall i j \in \Omega^{b r} \\
& \left(s_{i j, t}-1\right) B \leq u_{i, t}-u_{j, t}-2\left(r_{i j} P_{i j, t}+x_{i j} Q_{i j, t}\right)+\left(r_{i j}^{2}+x_{i j}^{2}\right) l_{i j, t} \leq\left(1-s_{i j, t}\right) B, \quad \forall t, \forall i j \in \Omega^{b r} \\
& u_{j}^{\min } \leq u_{j, t} \leq u_{j}^{\max }, \quad \forall t, \forall j \in \Omega^{E l e} \\
& 0 \leq L_{e, j, t}^{c r t, P} \leq L_{e, j, t}^{P}, \quad \forall t, \forall j \in \Omega^{E l e} \\
& 0 \leq L_{e, j, t}^{c r t, Q} \leq L_{e, j, t}^{Q}, \quad \forall t, \forall j \in \Omega^{E l e} \\
& L_{e, j, t}^{P} L_{e, j, t}^{c r t, Q}-L_{e, j, t}^{Q} L_{e, j, t}^{c r t, P}=0, \quad \forall t, \forall j \in \Omega^{E l e}
\end{aligned}
$$

Eq. (4) and (5) limit the active and reactive power from the upper grid. Eq. (6) denotes the limits of renewable energy output. Eq. (7) and (8) limit the range of the charging and discharging power of batteries, since we have considered the depreciation cost of batteries in the objective (1), the charging and discharging power of one battery will not be greater than zero simultaneously [12]. Eq. (9) represents the relationship between the stored energy in batteries and their charging/discharging power. Eq. (10) denotes the limits of the stored energy in batteries. Eq. (11) ensures that the stored energy in one battery at the last hour equals to that at the first hour in the scheduling time horizon [17], to guarantee the electricity adequacy after the contingency event. Eq. (12) limits the range of electric power of gas-fired generators. Eq. (13) refers to the electric power of gas-fired generators depends on their gas consumption. Eq. (14) limits the range of electricity supply to electric boilers. Eq. (15) denotes the bounds of the reactive power of compensators. Eq. 
(16)-(18) limit the range of active and reactive power flow in electric branches. Eq. (19) refers to the relationship between the power flow in branches and the nodal voltage. Eq. (20) denotes the bounds of the voltage at each bus. Eq. (21) and (22) limit the range of active and reactive load shedding. Eq. (23) denotes the active and reactive load shedding of an electric consumer should have a constant power factor [26].

The power flow model is non-convex and nonlinear due to (16). Since the electricity distribution systems usually are radial networks, the second-order cone relaxation is an effective method to deal with the non-convex constraint [25]. Thus, (16) can be replaced by the inequality constraint (24):

$$
\sqrt{\left(2 P_{i j, t}\right)^{2}+\left(2 Q_{i j, t}\right)^{2}+\left(u_{i, t}-l_{i j, t}\right)^{2}} \leq u_{i, t}+l_{i j, t}
$$

Although (24) is convex, it is still nonlinear. Therefore, we further employ the polyhedral approximation to replace (24) with a set of linear constraints [8]. Firstly, (24) can be divided into two conic quadratic constraints (25) and (26):

$$
\begin{gathered}
\sqrt{\left(2 P_{i j, t}\right)^{2}+\left(2 Q_{i j, t}\right)^{2}} \leq R_{i j, t} \\
\sqrt{\left(R_{i j, t}\right)^{2}+\left(u_{i, t}-l_{i j, t}\right)^{2}} \leq u_{i, t}+l_{i j, t}
\end{gathered}
$$

Since (25) is a conic quadratic constraint of dimension 2, it can be approximated with the linear constraints [27]:

$$
\begin{aligned}
& \left\{\begin{array}{l}
\xi^{0} \geq\left|2 P_{i, t,}\right| \\
\psi^{0} \geq\left|2 Q_{i j, t}\right|
\end{array}\right. \\
& \left\{\begin{array}{l}
\xi^{\sigma}=\cos \left(\frac{\pi}{2^{\sigma+1}}\right) \xi^{\sigma-1}+\sin \left(\frac{\pi}{2^{\sigma+1}}\right) \psi^{\sigma-1} \\
\psi^{\sigma} \geq\left|-\sin \left(\frac{\pi}{2^{\sigma+1}}\right) \xi^{\sigma-1}+\cos \left(\frac{\pi}{2^{\sigma+1}}\right) \psi^{\sigma-1}\right|, \quad \sigma=1, \ldots, v
\end{array}\right. \\
& \left\{\begin{array}{l}
\xi^{v} \leq R_{i, t} \\
\psi^{v} \leq \tan \left(\frac{\pi}{2^{v+1}}\right) \xi^{v}
\end{array}\right.
\end{aligned}
$$

In (27), we can adjust the parameter $v$ to control the error of polyhedral approximation, and the detailed analysis can be found in [8]. The approximation constraints of (26) are as similar as (27). Through the above approximations, the non-convex and nonlinear power flow model is converted to an elegant linear model, which can reduce the computational complexity of the post-contingency dispatch model.

\subsection{The natural gas system constraints}

In this paper, the steady-state natural gas flow model based on the Weymouth equation is employed to depict the gas system constraints, which is widely used in the planning and operation of IES [28]. Furthermore, considering the structure of gas systems and impacts of contingencies, the failures of gas sources, pipelines and compressors are considered in gas system constraints, as follows:

$$
\sum_{z \in \Omega_{m}^{G S}} G_{z, t}^{G S}+\sum_{n \in \Omega_{m}^{G a s}} G_{m n, t}+\sum_{\kappa \in \Omega_{d i s, m}^{C C,}} G_{d i s, \kappa, t}^{G C}-\sum_{\kappa \in \Omega_{s l c, m}^{G C}} G_{s u c, \kappa, t}^{G C}-\sum_{v \in \Omega_{m}^{G E}} G_{v, t}^{G E}-\sum_{d \in \Omega_{m}^{G B}} G_{d, t}^{G B}=L_{g, m, t}-L_{g, m, t}^{c r t}, \quad \forall t, \forall m \in \Omega^{G a s}
$$




$$
\begin{array}{ll}
s_{z, t}^{G S} G_{z}^{G S, \min } \leq G_{z, t}^{G S} \leq s_{z, t}^{G S} G_{z}^{G S, \max }, & \forall t, \forall z \in \Omega^{G S} \\
G_{m n, t}=\operatorname{sgn}\left(\Pi_{m, t}, \Pi_{n, t}\right) \eta_{m n} \sqrt{\left|\Pi_{m, t}^{2}-\Pi_{n, t}^{2}\right|}, \quad \text { if } s_{m n, t}=1 \\
-s_{m n, t} G_{m n}^{\max } \leq G_{m n, t} \leq s_{m n, t} G_{m n}^{\max }, & \forall t, \forall m n \in \Omega^{G P} \\
G_{d i s, \kappa, t}^{G C}=G_{s u c, \kappa, t}^{G C}\left(1-\eta_{\kappa}^{G C}\right), & \forall t, \forall \kappa \in \Omega^{G C} \\
s_{\kappa, t}^{G C} G_{\kappa}^{G C, \min } \leq G_{s u c, \kappa, t}^{G C} \leq s_{\kappa, t}^{G C} G_{\kappa}^{G C, \max }, & \forall t, \forall \kappa \in \Omega^{G C} \\
G_{v}^{G E, \min } \leq G_{v, t}^{G E} \leq G_{v}^{G E, \max }, & \forall t, \forall v \in \Omega^{G E} \\
G_{d}^{G B, \min } \leq G_{d, t}^{G B} \leq G_{d}^{G B, \max }, & \forall t, \forall d \in \Omega^{G B} \\
\Pi_{d i s, \kappa, t}^{G C} \leq \Phi_{\kappa}^{\max } \Pi_{s u c, \kappa, t}^{G C}, & \forall t, \forall \kappa \in \Omega^{G C}, \text { if } s_{\kappa, t}^{G C}=1 \\
\Pi_{m}^{\min } \leq \Pi_{m, t} \leq \Pi_{m}^{\max }, & \forall t, \forall m \in \Omega^{G a s} \\
0 \leq L_{g, m, t}^{c r t} \leq L_{g, m, t}, & \forall t, \forall m \in \Omega^{G a s}
\end{array}
$$

where $\Omega_{m}^{G S}$ and $\Omega_{m}^{G a s}$ denote the set of gas sources and gas nodes connected to node $m . \Omega_{\text {suc, } m}^{G C}$ and $\Omega_{d i s, m}^{G C}$ are the set of gas compressors leaving and entering node $m . \Omega_{m}^{G E}$ and $\Omega_{m}^{G B}$ are the set of gas-fired generators and gas boilers connected to node $m, \operatorname{sgn}(\cdot)$ is the sign function.

Eq. (28) denotes the gas balance on each gas node. Eq. (29) limits the output of gas sources considering the failures of gas sources. Eq. (30) and (31) denote the direction of gas flow in pipelines depends on the pressures of their connected nodes. Eq. (32) represents the gas consumption to run gas compressors [3]. Eq. (33) denotes the limits of transportation capacity of compressors. The limits of gas supply to gas-fired generators and gas boilers are (34) and (35). Eq. (36) denotes that the gas pressures of the inlet nodes and outlet nodes of compressors are limited by the maximal compression ratio. The nodal gas pressures are limited in (37). Eq. (38) denotes the limits of gas load shedding.

In the gas system constraints, the Weymouth equation (30) is non-convex and nonlinear. In the planning and operation of IES, an outer approximation approach based on Taylor series expansion around fixed pressure points is widely used to linearize the Weymouth equation [29, 30], which can effectively reduce the computational complexity of gas flow models. If the direction of gas flow in the pipeline $m n$ is from node $m$ to node $n$, we can use the following linear inequalities (39) to replace (30):

$$
G_{m n, t} \leq \eta_{m n}\left(\frac{\Pi_{m}^{\tau} \times \Pi_{m, t}}{\sqrt{\left(\Pi_{m}^{\tau}\right)^{2}-\left(\Pi_{n}^{\tau}\right)^{2}}}-\frac{\Pi_{n}^{\tau} \times \Pi_{n, t}}{\sqrt{\left(\Pi_{m}^{\tau}\right)^{2}-\left(\Pi_{n}^{\tau}\right)^{2}}}\right), \quad \forall t, \forall m n \in \Omega^{G P}, \forall\left(\Pi_{m}^{\tau}, \Pi_{n}^{\tau}\right) \in \Omega_{m n}^{F i x}
$$

where $\Omega_{m n}^{F i x}$ is the set of fixed pressure points $\left(\Pi_{m}^{\tau}, \Pi_{n}^{\tau}\right)$. These fixed points are generated by choosing multiple values from the gas pressure bounds of node $m$ and node $n$. Generally, using around 20 pairs of fixed points can provide a sufficient approximation of the Weymouth equation [31]. 
Furthermore, considering the gas flow in a pipeline can be bidirectional, two new variables $G_{m n, t}^{+}, G_{m, t}^{-}$and a binary variable $\delta_{m, t}$ are introduced, and Weymouth equation (30) is finally replaced by a set of constraints [29], as follows:

$$
\begin{gathered}
G_{m n, t}=G_{m n, t}^{+}-G_{m n, t}^{-}, \quad \forall t, \forall m n \in \Omega^{G P} \\
0 \leq G_{m n, t}^{+} \leq \delta_{m n, t} G_{m n}^{\max }, \quad \forall t, \forall m n \in \Omega^{G P} \\
0 \leq G_{m n, t}^{-} \leq\left(1-\delta_{m n, t}\right) G_{m n}^{\max }, \quad \forall t, \forall m n \in \Omega^{G P} \\
G_{m n, t}^{+} \leq \eta_{m n}\left(\frac{\Pi_{m}^{\tau} \times \Pi_{m, t}}{\sqrt{\left(\Pi_{m}^{\tau}\right)^{2}-\left(\Pi_{n}^{\tau}\right)^{2}}}-\frac{\Pi_{n}^{\tau} \times \Pi_{n, t}}{\sqrt{\left(\Pi_{m}^{\tau}\right)^{2}-\left(\Pi_{n}^{\tau}\right)^{2}}}\right)+\left(1-\delta_{m n, t}\right) B, \quad \forall t, \forall m n \in \Omega^{G P}, \forall\left(\Pi_{m}^{\tau}, \Pi_{n}^{\tau}\right) \in \Omega_{m n}^{F i x} \\
G_{m n, t}^{-} \leq \eta_{m n}\left(\frac{\Pi_{n}^{\tau *} \times \Pi_{n, t}}{\sqrt{\left(\Pi_{n}^{\tau *}\right)^{2}-\left(\Pi_{m}^{\tau *}\right)^{2}}}-\frac{\Pi_{m}^{\tau *} \times \Pi_{m, t}}{\sqrt{\left(\Pi_{n}^{\tau *}\right)^{2}-\left(\Pi_{m}^{\tau *}\right)^{2}}}\right)+\delta_{m n, t} B, \quad \forall t, \forall m n \in \Omega^{G P}, \forall\left(\Pi_{m}^{\tau *}, \Pi_{n}^{\tau *}\right) \in \Omega_{m n}^{F i x *}
\end{gathered}
$$

where $G_{m n, t}^{+}$denotes the gas flow from node $m$ to node $n$ at time $t$, and $G_{m n, t}^{-}$denotes the gas flow from $n$ to $m$. Since the gas pressure bounds of node $m$ and node $n$ may be different, we choose different fixed pressure points $\left(\Pi_{m}^{\tau}, \Pi_{n}^{\tau}\right)$ and $\left(\Pi_{m}^{\tau *}, \Pi_{n}^{\tau *}\right)$ in (43) and (44), respectively.

Compared to the piecewise linearization approach which is widely used to approximate the Weymouth equation [6], the approximation approach based on Taylor series expansion generates fewer binary variables (only one binary variable for one pipeline at each time interval), thereby effectively reducing the computational complexity.

\subsection{The heating system constraints}

A heating system usually contains a supply pipeline network and a return network. The typical hydraulic-thermal model of heating systems is non-convex and nonlinear [32], which is difficult to solve. Therefore, in the previous studies on the operation and market of IES, the constant-flow mode of heating networks is widely used, which assumes the mass flow rates in heating pipelines keep constant in the entire scheduling time horizon [8]. However, due to the impacts of contingencies, the topology of heating networks may be changed, which means the constant-flow assumption may be infeasible in the post-contingency dispatch of IES.

According to the studies on the reliability evaluation of district heating systems [33, 34], the topology changes of heating networks due to component failures mainly influence the hydraulic characteristics, and [20] shows that the mass flow rates have few impacts on the heat losses in pipelines. Therefore, in this paper, we employ the variable flow and constant temperature model in [35] to depict the constraints of heating systems considering component failures, which assumes the nodal temperatures are fixed and the mass flow rates can be adjusted. The heating system constraints are (45)-(59). (Besides, we can also use the more detailed variable flow and variable temperature model in post-contingency dispatch and linearize it with the piecewise McCormick relaxation [36]. However, although this model is more accurate, it is also more time-consuming.) 


$$
\begin{aligned}
& \sum_{c \in \Omega_{a}^{C F B}} H_{c, t}^{C F B}+\sum_{w \in \Omega_{a}^{E B}} H_{w, t}^{E B}+\sum_{d \in \Omega_{a}^{C B}} H_{d, t}^{G B}+H_{i n j, a, t}=L_{h, a, t}-L_{h, a, t}^{c r t}, \quad \forall t, \forall a \in \Omega^{\text {Heat }} \\
& H_{i n j, a, t}=\varsigma\left(\sum_{b \in f(a)} M_{b a, t}^{s p}-\sum_{b \in t(a)} M_{a b, t}^{s p}\right) \times\left(T_{a, t}^{s p}-T_{a, t}^{r t}\right) \\
& \eta_{a b}^{s p} M_{a b, t}^{s p}\left|M_{a b, t}^{s p}\right|=\Gamma_{a, t}^{s p}-\Gamma_{b, t}^{s p}, \quad \forall t, \forall a b \in \Omega_{H P}^{s p}, \text { if } s_{a b, t}^{s p}=1 \\
& \eta_{a b}^{r t} M_{a b, t}^{r t}\left|M_{a b, t}^{r t}\right|=\Gamma_{a, t}^{r t}-\Gamma_{b, t}^{r t}, \quad \forall t, \forall a b \in \Omega_{H P}^{r t}, \text { if } s_{a b, t}^{r t}=1 \\
& \left(\sum_{b \in f(a)} M_{b a, t}^{s p}-\sum_{b \in t(a)} M_{a b, t}^{s p}\right)+\left(\sum_{b \in f(a)} M_{b a, t}^{r t}-\sum_{b \in t(a)} M_{a b, t}^{r t}\right)=0 \\
& s_{c, t}^{C F B} H_{c}^{C F B, \text { min }} \leq H_{c, t}^{C F B} \leq s_{c, t}^{C F B} H_{c}^{C F B, \max }, \forall t, \forall c \in \Omega^{C F B} \\
& H_{w, t}^{E B}=\eta_{w}^{E B} P_{w, t}^{E B}, \quad \forall t, \forall w \in \Omega^{E B} \\
& s_{w, t}^{E B} H_{w}^{E B, \min } \leq H_{w, t}^{E B} \leq S_{w, t}^{E B} H_{w}^{E B, \max }, \quad \forall t, \forall w \in \Omega^{E B} \\
& H_{d, t}^{G B}=\eta_{d}^{G B} G_{d, t}^{G B} \times G H V, \quad \forall t, \forall d \in \Omega^{G B} \\
& s_{d, t}^{G B} H_{d}^{G B, \min } \leq H_{d, t}^{G B} \leq s_{d, t}^{G B} H_{d}^{G B, \max }, \quad \forall t, \forall d \in \Omega^{G B} \\
& -s_{a b, t}^{s p} M_{a b}^{s p, \max } \leq M_{a b, t}^{s p} \leq s_{a b, t}^{s p} M_{a b}^{s p, \max }, \quad \forall t, \forall a b \in \Omega_{H P}^{s p} \\
& -s_{a b, t}^{r t} M_{a b}^{r t, \max } \leq M_{a b, t}^{r t} \leq s_{a b, t}^{r t} M_{a b}^{r t, \max }, \quad \forall t, \forall a b \in \Omega_{H P}^{r t} \\
& \Gamma_{a}^{s p, \min } \leq \Gamma_{a, t}^{s p} \leq \Gamma_{a}^{s p, \max }, \quad \forall t, \forall a \in \Omega^{\text {Heat }} \\
& \Gamma_{a}^{r t, \min } \leq \Gamma_{a, t}^{r t} \leq \Gamma_{a}^{r t, \max }, \quad \forall t, \forall a \in \Omega^{\text {Heat }} \\
& 0 \leq L_{h, a, t}^{c r t} \leq L_{h, a, t}, \quad \forall t, \forall a \in \Omega^{\text {Heat }}
\end{aligned}
$$

where $\Omega_{a}^{C F B}, \Omega_{a}^{E B}$ and $\Omega_{a}^{G B}$ denote the set of coal-fired boilers, electric boilers and gas boilers connected to the node $a$.

In heating system constraints, (45) denotes the nodal heating power balance, (46) represents the injected heating power of a node depends on the injected mass flow and the nodal temperatures, which interlinks the hydraulic model and thermal model. Besides, based on the aforementioned constant temperature assumption, the fixed temperatures $T_{a, t}^{s p}$ and $T_{a, t}^{r t}$ can be treated as constants in (46). Eq. (47) and (48) denote the mass flow in one pipeline depends on the pressures of connected nodes. Eq. (49) denotes the continuity of mass flow, which means the mass flow that enters a node in the supply network should equal to the mass flow that leaves the node in the return network. Eq. (50) denotes the limits of heat power of coal-fired boilers. Eq. (51) denotes the relationship of the heat power and electricity demand of electric boilers, and (52) represents the limits of heat power of electric boilers. Eq. (53) denotes the relationship of the heat power and gas consumption of gas boilers, and (54) denotes the limits of heat power of gas boilers. Eq. (55) and (56) denote the bounds of the mass flow in pipelines. Eq. (57) and (58) denote the limits of nodal pressures. Eq. (59) limits the heat load shedding.

In heating system constraints, the mass flow equations (47) and (48) are non-convex and nonlinear, which are 
similar to gas flow equations (30) and (31). Thus, we can introduce two new variables $M_{a b, t}^{s p+}, M_{a b, t}^{s p-}$ and a binary variable $\delta_{a b, t}^{s p}$, then approximate the mass flow equation (47) with the Taylor series expansion, as follows:

$$
\begin{array}{cc}
M_{a b, t}^{s p}=M_{a b, t}^{s p+}-M_{a b, t}^{s p-}, & \forall t, \forall a b \in \Omega_{H P}^{s p} \\
0 \leq M_{a b, t}^{s p+} \leq \delta_{a b, t}^{s p} M_{a b}^{s p, \max }, & \forall t, \forall a b \in \Omega_{H P}^{s p} \\
0 \leq M_{a b, t}^{s p-} \leq\left(1-\delta_{a b, t}^{s p}\right) M_{a b}^{s p, \max }, & \forall t, \forall a b \in \Omega_{H P}^{s p} \\
M_{a b, t}^{s p+} \leq \frac{1}{2 \sqrt{\eta_{a b}^{s p}}} \times \frac{\left(\Gamma_{a, t}^{s p}-\Gamma_{b, t}^{s p}\right)+\left(\Gamma_{a}^{s p, \tau}-\Gamma_{b}^{s p, \tau}\right)}{\sqrt{\Gamma_{a}^{s p, \tau}-\Gamma_{b}^{s p, \tau}}}+\left(1-\delta_{a b, t}^{s p}\right) B, & \forall t, \forall a b \in \Omega_{H P}^{s p}, \forall\left(\Gamma_{a}^{s p, \tau}, \Gamma_{b}^{s p, \tau}\right) \in \Omega_{a b}^{s p, F i x} \\
M_{a b, t}^{s p-} \leq \frac{1}{2 \sqrt{\eta_{a b}^{s p}}} \times \frac{\left(\Gamma_{b, t}^{s p}-\Gamma_{a, t}^{s p}\right)+\left(\Gamma_{b}^{s p, \tau *}-\Gamma_{a}^{s p, \tau *}\right)}{\sqrt{\Gamma_{b}^{s p, \tau *}-\Gamma_{a}^{s p, \tau *}}+\delta_{a b, t}^{s p} B,} & \forall t, \forall a b \in \Omega_{H P}^{s p}, \forall\left(\Gamma_{a}^{s p, \tau *}, \Gamma_{b}^{s p, \tau *}\right) \in \Omega_{a b}^{s p, F i x *}
\end{array}
$$

where $M_{a b, t}^{s p+}$ denotes the mass flow from node $a$ to $b$ at time $t$, while $M_{a b, t}^{s p-}$ represents the mass flow from node $b$ to $a . \delta_{a b, t}^{s p}$ is a binary variable which denotes the actual direction of mass flow. $\left(\Gamma_{a}^{s p, \tau}, \Gamma_{b}^{s p, \tau}\right)$ and $\left(\Gamma_{a}^{s p, \tau^{*}}, \Gamma_{b}^{s p, \tau^{*}}\right)$ are fixed pressure points chosen from the pressure bounds of node $a$ and node $b$. Based on these approximations, we can linearize the non-convex and nonlinear constraint (47). For (48), the approximation method is similar.

\section{The distributed emergency energy trading algorithm for the IES}

In Section 3, we present the post-contingency dispatch model of the IES in a centralized formulation. As previously mentioned, in the IES with multiple participants, each participant will make post-contingency dispatch decisions independently and the coordination of different participants is achieved via energy trading. Therefore, considering the independence of participants, a distributed emergency energy trading algorithm for the IES is developed in this section, in order to efficiently solve the post-contingency dispatch model of IES in a decentralized manner.

In the proposed emergency energy market framework of IES, a distributed price-directed optimization mechanism is essential, which can effectively coordinate different participants with individual profit goals. Thus, in this paper, the ADMM is employed to develop a distributed emergency energy trading algorithm for IES. ADMM is a distributed convex optimization method that uses the dual price adjustment [37]. The dual price adjustment is similar to the Walrasian auction, where the trading prices of goods are adjusted according to the differences of demand and supply [12]. The trading prices will increase when the supply is insufficient to satisfy the demand and the prices will decrease when the supply exceeds the demand, finally achieving the balance of supply and demand. With the ADMM, we can easily calculate the optimal emergency energy trading prices, thereby coordinating the energy allocation among participants. Furthermore, to enhance the convergence speed of the algorithm, the ADMM with self-adaptive penalty parameters [38] is employed in this paper. 
Firstly, to use the distributed energy trading algorithm, it is essential to construct the coupling constraints (65)-(67):

$$
\begin{array}{ll}
P_{w, t}^{E B, D}=P_{w, t}^{E B, S}, & \forall t, \forall w \in \Omega^{E B} \\
G_{v, t}^{G E, D}=G_{v, t}^{G E, S}, & \forall t, \forall v \in \Omega^{G E} \\
G_{d, t}^{G B, D}=G_{d, t}^{G B, S}, & \forall t, \forall d \in \Omega^{G B}
\end{array}
$$

where $P_{w, t}^{E B, D}$ denotes the electricity demand of electric boiler $w$ at time $t$ which is decided by HSO, $P_{w, t}^{E B, S}$ is the electricity supply to electric boiler $w$ decided by ESO. $G_{v, t}^{G E, D}$ is the gas demand of gas-fired generator $v$ decided by ESO, $G_{v, t}^{G E, S}$ is the gas supply to generator $v$ decided by GSO. $G_{d, t}^{G B, D}$ is the gas demand of gas boiler $d$ decided by HSO, $G_{d, t}^{G B, S}$ is the gas supply to gas boiler $d$ decided by GSO.

Then, we can use $P_{w, t}^{E B, S}$ and $G_{v, t}^{G E, D}$ to replace $P_{w, t}^{E B}$ and $G_{v, t}^{G E}$ in electricity system constraints, respectively. In gas system constraints, we can use $G_{v, t}^{G E, S}$ and $G_{d, t}^{G B, S}$ to replace $G_{v, t}^{G E}$ and $G_{d, t}^{G B}$. And in heating system constraints, $P_{w, t}^{E B}$ and $G_{d, t}^{G B}$ can be replaced by $P_{w, t}^{E B, D}$ and $G_{d, t}^{G B, D}$. Afterward, according to the ADMM in [38], the distributed emergency energy trading algorithm is developed. The pseudocode is presented in Algorithm 1, and the detailed procedures are as follows:

Algorithm 1: ADMM for distributed emergency energy trading

1: Initialize the coupling variables, emergency energy prices, and penalty parameters. Set the iteration index $k=0$, the threshold of primal and dual residuals $\bar{\varepsilon}_{\text {pri }}$ and $\bar{\varepsilon}_{\text {dual }}$.

2: Each participant shares the information of its coupling variables with each other. Then, calculate the average values of coupling variables according to (68)-(70).

3: Each participant solves its post-contingency dispatch model in parallel:

3.1) ESO solves its local optimization model (71)

3.2) GSO solves its local optimization model (72)

3.3) HSO solves its local optimization model (73)

Then update the average values of coupling variables.

4: EETP calculates the primal and dual residuals according to (74).

If the primal and dual residuals are less than the given threshold, the algorithm achieves convergence, go to Step 5.

Otherwise, set $k=k+1$, EETP adjusts the penalty parameters $\rho_{w}^{E B(k+1)}, \rho_{v}^{G E(k+1)}$ and $\rho_{d}^{G B(k+1)}$ according to (75), and updates the emergency energy prices $\lambda_{w, t}^{E B(k+1)}, \lambda_{v, t}^{G E(k+1)}$ and $\lambda_{d, t}^{G B(k+1)}$ according to (76)-(78). Then, repeat Step 3-4.

5: Emergency energy trading settlement.

Step 1: Initialization. Once a contingency event occurs, the distributed emergency energy trading among 
participants will be activated. Then, set the iteration index $k=0$. Initialize the coupling variables $P_{w, t}^{E B, D(0)}, P_{w, t}^{E B, S(0)}$, $G_{v, t}^{G E, D(0)}, G_{v, t}^{G E, S(0)}, G_{d, t}^{G B, D(0)}$ and $G_{d, t}^{G B, S(0)}$. Set the initial value of emergency energy prices $\lambda_{w, t}^{E B(0)}, \lambda_{v, t}^{G E(0)}$ and $\lambda_{d, t}^{G B(0)}$. Initialize the penalty parameters $\rho_{w}^{E B(0)}, \rho_{v}^{G E(0)}$ and $\rho_{d}^{G B(0)}$. And set the threshold of primal residuals and dual residuals $\bar{\varepsilon}_{p r i}$ and $\bar{\varepsilon}_{\text {dual }}$.

Step 2: Calculate the average values of coupling variables. Each participant shares the information of its coupling variables with other participants. Then, calculate the average values of coupling variables:

$$
\begin{array}{ll}
\tilde{P}_{w, t}^{E B(k)}=\frac{1}{2}\left(P_{w, t}^{E B, D(k)}+P_{w, t}^{E B, S(k)}\right), & \forall t, \forall w \in \Omega^{E B} \\
\tilde{G}_{v, t}^{G E(k)}=\frac{1}{2}\left(G_{v, t}^{G E, D(k)}+G_{v, t}^{G E, S(k)}\right), & \forall t, \forall v \in \Omega^{G E} \\
\tilde{G}_{d, t}^{G B(k)}=\frac{1}{2}\left(G_{d, t}^{G B, D(k)}+G_{d, t}^{G B, S(k)}\right), & \forall t, \forall d \in \Omega^{G B}
\end{array}
$$

Step 3: The EETP broadcasts emergency energy prices and penalty parameters to participants. Then, in parallel, each participant conducts post-contingency dispatch:

For ESO, it will update its local decision variables by solving the following augmented Lagrangian, subject to the electricity system constraints.

$$
\begin{aligned}
\min & \sum_{t=t_{\text {sart }}}^{t_{\text {end }}}\left\{C^{g r i d} P_{t}^{g r i d}+\sum_{y \in \Omega^{R E}} C_{y}^{R E} P_{y, t}^{R E}+\sum_{o \in \Omega^{E S S}} C_{o}^{E S S}\left(P_{c h, o, t}^{E S S}+P_{d c h, o, t}^{E S S}\right)+\sum_{j \in \Omega^{E l e}} C_{e, j}^{c r t} L_{e, j, t}^{c r t, P}\right. \\
& \left.+\sum_{w \in \Omega^{E B}}\left(\lambda_{w, t}^{E B(k)}\left(\tilde{P}_{w, t}^{E B(k)}-P_{w, t}^{E B, S}\right)+\frac{\rho_{w}^{E B(k)}}{2}\left\|\tilde{P}_{w, t}^{E B(k)}-P_{w, t}^{E B, S}\right\|_{2}^{2}\right)+\sum_{v \in \Omega^{G E}}\left(\lambda_{v, t}^{G E(k)}\left(G_{v, t}^{G E, D}-\tilde{G}_{v, t}^{G E(k)}\right)+\frac{\rho_{v}^{G E(k)}}{2}\left\|G_{v, t}^{G E, D}-\tilde{G}_{v, t}^{G E(k)}\right\|_{2}^{2}\right)\right\}
\end{aligned}
$$

subject to (2)-(15), (17)-(23), (27)

The objective function of ESO in (71) includes the electricity generation and storage cost, the economic cost of electric load shedding, the revenue and payment of emergency energy trading with other participants, and the penalty terms for violating the coupling constraints.

For GSO, it will solve the following local optimization model to update its decision variables:

$$
\begin{aligned}
& \min \sum_{t=t_{\text {sart }}}^{t_{\text {end }}}\left\{\sum_{z \in \Omega^{G S}} C_{z}^{G S} G_{z, t}^{G S}+\sum_{m \in \Omega^{G a s}} C_{g, m}^{c r t} L_{g, m, t}^{c r t}+\sum_{v \in \Omega^{G E}}\left(\lambda_{v, t}^{G E(k)}\left(\tilde{G}_{v, t}^{G E(k)}-G_{v, t}^{G E, S}\right)+\frac{\rho_{v}^{G E(k)}}{2}\left\|\tilde{G}_{v, t}^{G E(k)}-G_{v, t}^{G E, S}\right\|_{2}^{2}\right)\right. \\
& \left.\quad+\sum_{d \in \Omega^{G B}}\left(\lambda_{d, t}^{G B(k)}\left(\tilde{G}_{d, t}^{G B(k)}-G_{d, t}^{G B, S}\right)+\frac{\rho_{d}^{G B(k)}}{2}\left\|\tilde{G}_{d, t}^{G B(k)}-G_{d, t}^{G B, S}\right\|_{2}^{2}\right)\right\} \\
& \text { subject to (28), (29), (32)-(38), (40)-(44) }
\end{aligned}
$$

The objective function of GSO in (72) includes the supply cost of gas sources, the economic cost of gas load shedding, the revenue of selling natural gas to other participants, and the penalty terms for violating the coupling constraints. The constraints include the gas system constraints.

And HSO can solve the following local optimization model to conduct its post-contingency dispatch: 


$$
\begin{aligned}
& \min \sum_{t=t_{\text {satr }}}^{t_{\text {end }}}\left\{\sum_{c \in \Omega^{C F B}} C_{c}^{C F B} H_{c, t}^{C F B}+\sum_{a \in \Omega^{\text {Heat }}} C_{h, a}^{c r t} L_{h, a, t}^{c r t}+\sum_{w \in \Omega^{E B}}\left(\lambda_{w, t}^{E B(k)}\left(P_{w, t}^{E B, D}-\tilde{P}_{w, t}^{E B(k)}\right)+\frac{\rho_{w}^{E B(k)}}{2}\left\|P_{w, t}^{E B, D}-\tilde{P}_{w, t}^{E B(k)}\right\|_{2}^{2}\right)\right. \\
& \left.\quad+\sum_{d \in \Omega^{G B}}\left(\lambda_{d, t}^{G B(k)}\left(G_{d, t}^{G B, D}-\tilde{G}_{d, t}^{G B(k)}\right)+\frac{\rho_{d}^{G B(k)}}{2}\left\|G_{d, t}^{G B, D}-\tilde{G}_{d, t}^{G B(k)}\right\|_{2}^{2}\right)\right\}
\end{aligned}
$$

subject to (45), (46), (49)-(54), (57)-(64)

The objective function of HSO in (73) contains the operation cost of coal-fired boilers, the economic cost of heat load shedding, the payment of purchasing electricity and gas from other participants, as well as the penalty terms. The constraints are heating system constraints.

After each participant solves its local optimization, participants will share the latest values of its coupling variables with each other. Then, update the average values of coupling variables $\tilde{P}_{w, t}^{E B(k+1)}, \tilde{G}_{v, t}^{G E(k+1)}$ and $\tilde{G}_{d, t}^{G B(k+1)}$.

Step 4: According to the dispatch decisions of Step 3, each participant submits the latest information of its energy supply/demand plan (the coupling variables $P_{w, t}^{E B, S(k+1)}, P_{w, t}^{E B, D(k+1)}, G_{v, t}^{G E, S(k+1)}, G_{v, t}^{G E, D(k+1)}, G_{d, t}^{G B, S(k+1)}$ and $G_{d, t}^{G B, D(k+1)}$ ) to the EETP. Then EETP calculates the primal and dual residuals, and accordingly updates the emergency energy prices (dual prices) and penalty terms.

Take the electricity trading among ESO and HSO as an example, the primal and dual residuals are calculated as:

$$
\left\{\begin{array}{l}
\varepsilon_{\text {pri, }}^{E B(k+1)}=\max \left\{\left\|P_{w, t}^{E B, D(k+1)}-\tilde{P}_{w, t}^{E B(k+1)}\right\|_{2}, \forall t\right\} \\
\varepsilon_{\text {dual }, w}^{E B(k+1)}=\max \left\{\left\|\tilde{P}_{w, t}^{E B(k+1)}-\tilde{P}_{w, t}^{E B(k)}\right\|_{2}, \forall t\right\}
\end{array}, \quad \forall w \in \Omega^{E B}\right.
$$

For the energy trading among other participants, the calculation of primal and dual residuals is similar to (74). If the maximal value of all primal residuals is less than $\bar{\varepsilon}_{p r i}$ and the maximal value of dual residuals is less than $\bar{\varepsilon}_{\text {dual }}$, the algorithm achieves convergence, which means the demand of buyers and the supply of sellers balance. Then, go to Step 5.

Otherwise, the EETP will update the penalty parameters according to primal and dual residuals:

$$
\rho_{w}^{E B(k+1)}=\left\{\begin{array}{l}
\alpha^{i n c r} \rho_{w}^{E B(k)}, \quad \text { if } \varepsilon_{\text {pri,w }}^{E B(k+1)}>\mu \times \varepsilon_{\text {dual }, w}^{E B(k+1)} \\
\rho_{w}^{E B(k)} / \alpha^{\text {decr }}, \quad \text { if } \varepsilon_{d u a l, w}^{E B(k+1)}>\mu \times \varepsilon_{p r i, w}^{E B(k+1)} \\
\rho_{w}^{E B(k)}, \text { otherwise }
\end{array}\right.
$$

where $\alpha^{\text {incr }}>1, \alpha^{\text {decr }}>1$, and $\mu>1$ are parameters to adjust the update speed. For other penalty parameters $\rho_{v}^{G E(k+1)}$ and $\rho_{d}^{G B(k+1)}$, the update process is similar. The varying penalty parameters can improve the convergence speed of the algorithm in practice. Moreover, according to [37], to ensure the theoretical convergence of ADMM, we can fix the values of penalty parameters after a finite number of iterations.

Then, EETP can update the emergency energy prices as:

$$
\lambda_{w, t}^{E B(k+1)}=\lambda_{w, t}^{E B(k)}+\rho_{w}^{E B(k+1)}\left(P_{w, t}^{E B, D(k+1)}-\tilde{P}_{w, t}^{E B(k+1)}\right)
$$




$$
\begin{aligned}
& \lambda_{v, t}^{G E(k+1)}=\lambda_{v, t}^{G E(k)}+\rho_{v}^{G E(k+1)}\left(G_{v, t}^{G E, D(k+1)}-\tilde{G}_{v, t}^{G E(k+1)}\right) \\
& \lambda_{d, t}^{G B(k+1)}=\lambda_{d, t}^{G B(k)}+\rho_{d}^{G B(k+1)}\left(G_{d, t}^{G B, D(k+1)}-\tilde{G}_{d, t}^{G B(k+1)}\right)
\end{aligned}
$$

Then, go back to Step 3 .

Step 5: Emergency energy trading settlement. Once the emergency energy trading algorithm achieves convergence, the participants of IES will trade energy with each other obeying the values of coupling variables until the end of the contingency event. Meanwhile, the buyers need to pay energy purchase cost to sellers according to the emergency energy prices. Furthermore, each participant will calculate its operation cost during the contingency event.

In the emergency energy market of IES, if the uncertainties of renewable energy and loads are considered, we can use the receding horizon model predictive control method in [12] to ensure the effective cooperation of participants. The basic principle is that once the distributed energy trading between participants is finished for the current dispatch interval (e.g., $\left.t_{\text {start }}\right)$, the dispatch interval will recede by a step $\left(t_{\text {start }} \leftarrow t_{\text {start }}+1\right)$ and participants can update their generation and load predictions. Then, the coordinated post-contingency dispatch model of IES will be solved for the new scheduling time horizon. It can provide a real-time dispatch to deal with the uncertainties of renewable energy and loads, and the detailed algorithm can be found in [12].

Moreover, in this paper, we use 1 hour as the time step for the post-contingency dispatch models of different energy systems, which can effectively reduce the complexity for the coordinated dispatch problem of multiple energy systems and enhance computational tractability. If the difference in the time scale of electricity, gas and heating systems is considered in detail, we can modify the dispatch interval in the dispatch models of participants (71)-(73) and use the multi-time scale dispatch framework to deal with the difference, and one can refer to [39, 40] for the detailed approaches.

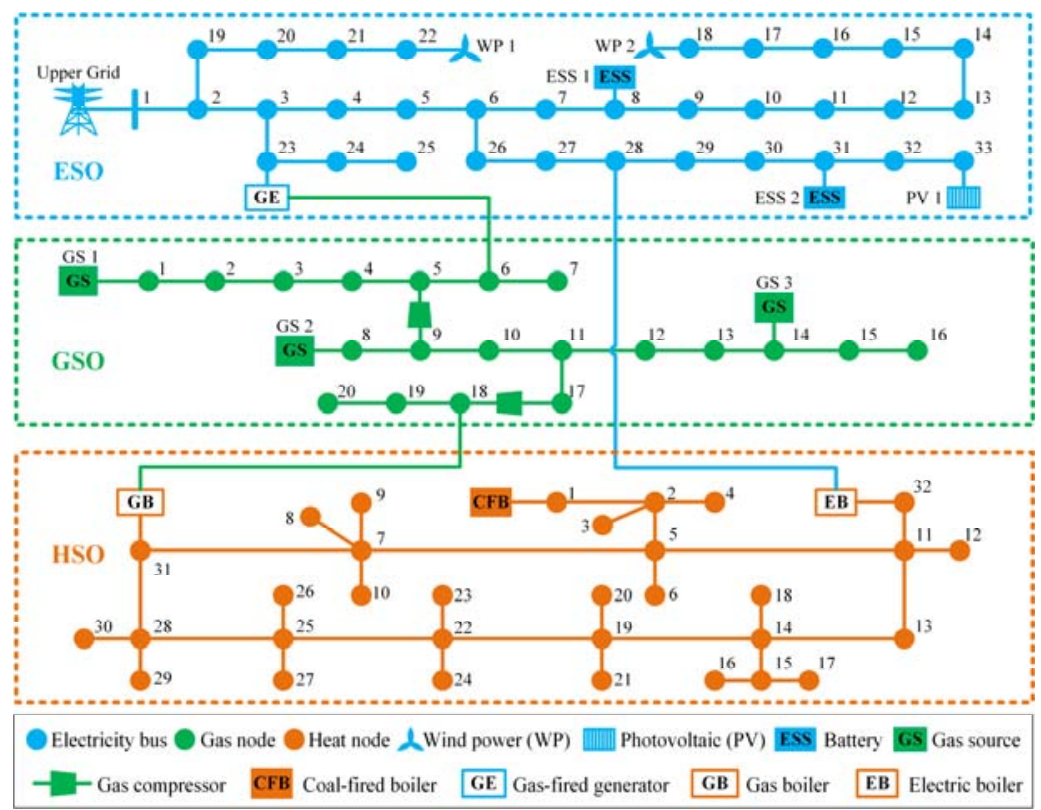


Fig. 4 The scheme of the IES test case

\section{Case studies}

To validate the effectiveness of the proposed post-contingency dispatch method, case studies are firstly conducted on an IES consist of an IEEE Case33 electricity system [41], a modified 20-node Belgium natural gas system [42] and a modified 32-node district heating system of Barry Island [32]. The electricity, gas and heating systems are respectively managed by one ESO, GSO and HSO. And the three systems are coupled via one gas-fired generator, gas boiler and electric boiler. The scheme of the IES is shown in Fig. 4.

The maximal total load of electric consumers is $3.655 \mathrm{MW}$, the maximal load of gas consumers is $50 \mathrm{kcf} / \mathrm{h}$, and the maximal load of heat consumers is $2.164 \mathrm{MW}$. The capacities of two wind power generations and one PV arrays (WP1, WP2 and PV1) are 1.5, 1.5 and $2 \mathrm{MW}$, respectively. The hourly profiles of loads and renewable energy generation in a sample day from [21] and [14] are shown in Fig. 5 and Fig. 6. The initial values of penalty parameters are all set to 5 and the tolerances of primal and dual residuals are set to 0.001. The data of three energy networks can be found in [32], [41], and [42]. Other system configuration data (e.g., the parameters of energy coupling components and the values of fixed pressure points) can be found in [43]. Case studies are solved by Matlab R2014a and Gurobi 9.0 on a personal computer with 16 GB RAM.

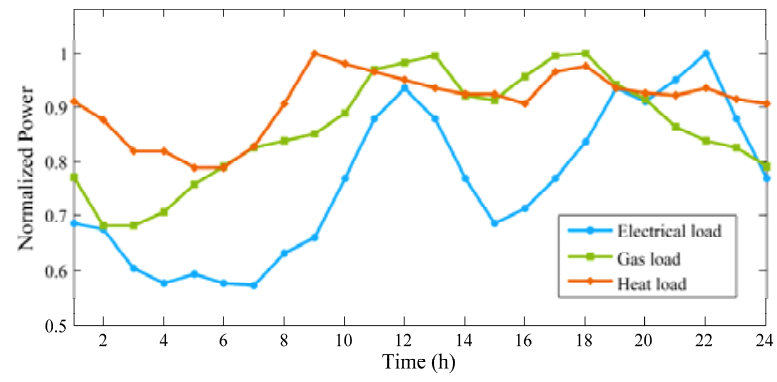

Fig. 5 The normalized hourly load profiles

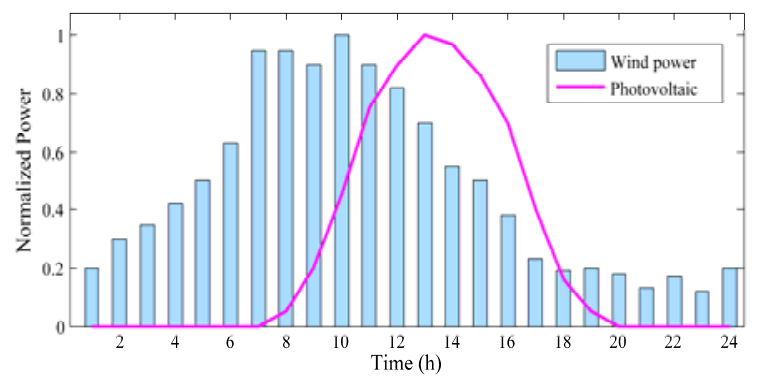

Fig. 6 The normalized hourly profiles of renewable energy

To demonstrate the effectiveness and practicability of the proposed method, case studies are divided into four parts. In the first part, the proposed method is compared with the traditional centralized post-contingency dispatch method, which validates the effectiveness of the proposed method in optimizing the emergency energy dispatch of the entire IES. In the second part, the proposed method is compared with another decentralized method based on regulated energy trading prices, which validates the advantages of the proposed method in fully stimulating the coordination of different participants. In the third part, we compare the proposed model and algorithm with other energy network models and distributed optimization approaches, thereby demonstrating the accuracy and computational efficiency of the proposed method. In the fourth part, a larger scale IES is employed to show the application of the post-contingency dispatch 
method in practice.

\subsection{Comparison of the proposed post-contingency dispatch method with the centralized method}

In this part, the proposed method based on distributed energy trading is compared with the traditional centralized dispatch method. Firstly, the following cases are defined:

Case 1: Due to the failure of the upper grid, the electricity supply from the upper grid is interrupted from 1:00 to 12:00. To deal with this contingency, the proposed post-contingency dispatch method is employed.

Case 2: The contingency is the same as Case 1. However, the IES is managed by a centralized operator. Therefore, the centralized post-contingency dispatch method in [5] and [6] is used, whose results are used as the benchmark.

\subsubsection{The convergence of the proposed post-contingency dispatch method}

In Case 1, the convergence of the proposed post-contingency dispatch method is demonstrated in Fig. 7. As shown in Fig. 7, with the increase of iterations, the maximal values of primal and dual residuals gradually decrease, finally reaching the tolerance of gap 0.001 after 45 iterations. The proposed distributed energy trading algorithm takes $63.69 \mathrm{sec}$ to achieve convergence, which means it can provide the emergency dispatch strategies for IES in a short time.

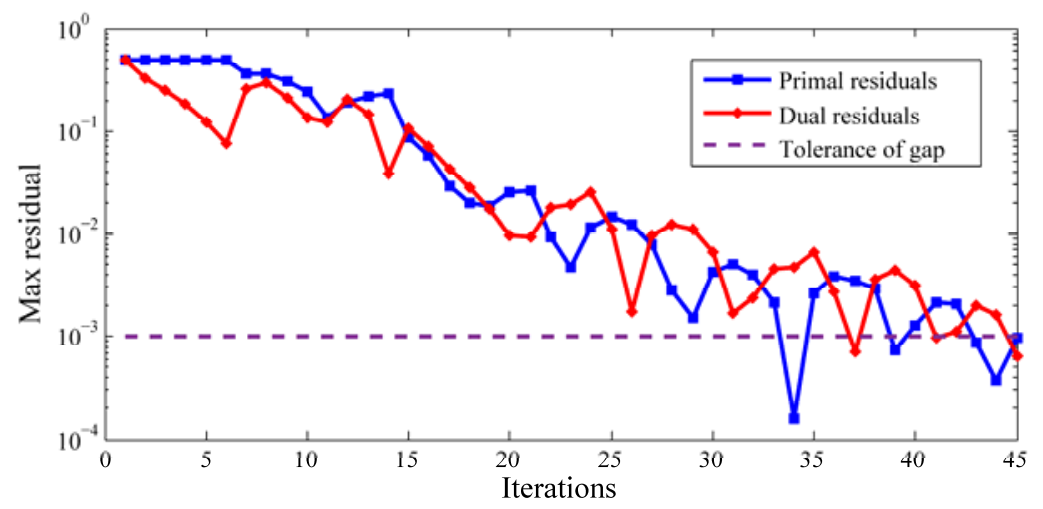

Fig. 7 Evolution of the maximal residuals

\subsubsection{The post-contingency dispatch results of Case 1 and Case 2}

Due to the failure of the upper grid, the electricity supply of ESO is limited. Therefore, in post-contingency dispatch, it is essential to adjust the energy allocation among ESO, GSO, and HSO, thereby minimizing the total load curtailment cost of IES. During this contingency, the power output results of energy coupling components (EB, GB and GE) in Case 1 and Case 2 are presented in Table 1.

As shown in Table 1, From 1:00 to 6:00, due to the lower available power of renewable energy, the electricity supply to EB is reduced and the electric power of GE is increased in two cases to minimize the load shedding of electric consumers. Meanwhile, the heat power of GB is fixed at its maximal power, thereby supplying the demand of heat consumers as much as possible. On the other hand, from 7:00 to 12:00, with the increase of renewable energy output 
(especially the output of PV), the electricity shortage of the ESO is in remission. Thus, the electricity supply to EB (the heat power of EB) is increased in Case 1 and Case 2. The results of Table 1 show that, during the contingency, the power outputs of EB and GB are same in Case 1 and Case 2. Moreover, although the hourly power profiles of GE have differences in Case 1 and Case 2, the total amount of its electricity output is very close in the two cases.

Table 1. The power output of energy coupling components (MW)

\begin{tabular}{ccccccc}
\hline \multirow{2}{*}{$\begin{array}{c}\text { Time } \\
\text { (hour) }\end{array}$} & \multicolumn{3}{c}{ Case 1 } & \multicolumn{3}{c}{ Case 2 } \\
\cline { 2 - 7 } & EB & GB & GE & EB & GB & GE \\
\hline 1 & 0.2798 & 0.9 & 0.5 & 0.2798 & 0.9 & 0.5 \\
2 & 0.3757 & 0.9 & 0.5 & 0.3757 & 0.9 & 0.5 \\
3 & 0.2572 & 0.9 & 0.5 & 0.2572 & 0.9 & 0.5 \\
4 & 0.2572 & 0.9 & 0.5 & 0.2572 & 0.9 & 0.5 \\
5 & 0.3709 & 0.9 & 0.5 & 0.3709 & 0.9 & 0.5 \\
6 & 0.3709 & 0.9 & 0.5 & 0.3709 & 0.9 & 0.5 \\
7 & 0.4563 & 0.9 & 0.4432 & 0.4563 & 0.9 & 0.2177 \\
8 & 0.6336 & 0.9 & 0.4474 & 0.6336 & 0.9 & 0.5 \\
9 & 0.8437 & 0.9 & 0.4522 & 0.8437 & 0.9 & 0.5 \\
10 & 0.7977 & 0.9 & 0.3176 & 0.7977 & 0.9 & 0.5 \\
11 & 0.7649 & 0.9 & 0.3176 & 0.7649 & 0.9 & 0.2596 \\
12 & 0.7320 & 0.9 & 0.3176 & 0.7320 & 0.9 & 0.3206 \\
Sum & 6.1399 & 10.8 & 5.2956 & 6.1399 & 10.8 & 5.2979 \\
\hline
\end{tabular}

Moreover, Fig. 8 and Fig. 9 present the power of electric and heat load shedding and the charging/discharging power of batteries for Case 1, respectively. From 1:00 to 6:00, due to the electricity supply of IES is in severe shortage, ESO has to curtail its electric load and the electricity supply to EB. It results in the heat power of EB is reduced and HSO also has to curtail the load of heat consumers. During this period, ESO discharges the stored energy of batteries to supply the important loads. From 7:00 to 12:00, since the electricity shortage of IES is in remission, both the electric load shedding and heat load shedding are reduced to 0 . Meanwhile, batteries are charged to recover their stored energy. In the postcontingency dispatch of IES, energy storage components can operate in discharge mode to supply important loads when the electricity supply is in shortage and they can store energy when the electricity supply is adequate, thereby enhancing the operational flexibility of the IES.
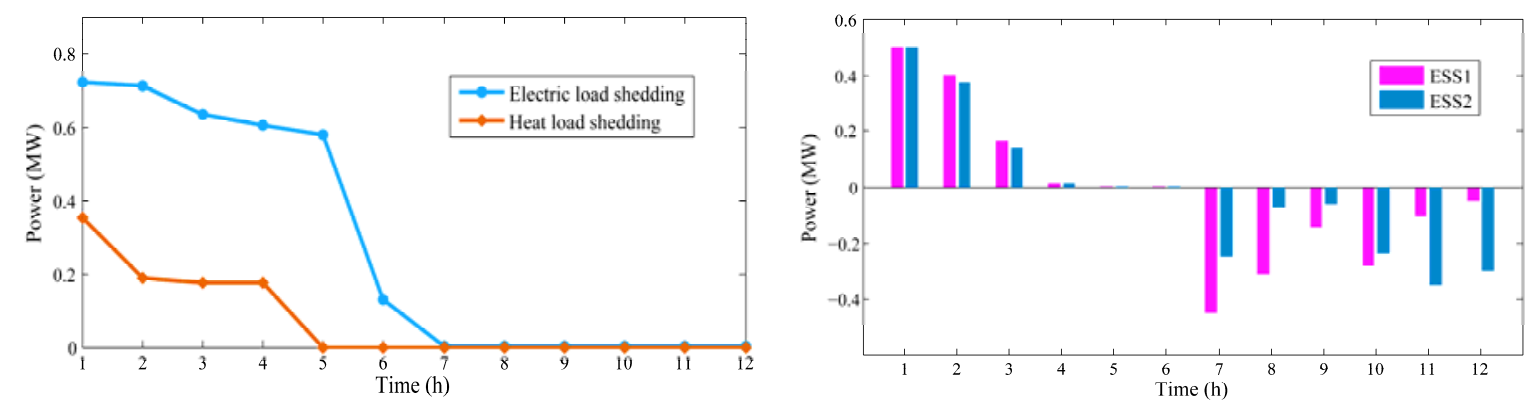
Fig. 8 The power of electric and heat load shedding

Fig. 9 The charging and discharging power of batteries

Table 2 presents the post-contingency dispatch results of the IES in two cases. The results validate the proposed post-contingency dispatch method can effectively optimize the total operation cost and load curtailment cost of IES during the contingency event, as same as the centralized dispatch method. Moreover, with the proposed method, each participant can make dispatch decisions independently, which can protect the data privacy of participants.

Table 2. The post-contingency dispatch results of Case 1 and Case 2

\begin{tabular}{lcc}
\hline Cost $(\$)$ & Case 1 & Case 2 \\
\hline Total operation cost of IES & 22842.55 & 22842.55 \\
Total load curtailment cost & 907.33 & 907.33 \\
Electric load curtailment cost & 676.60 & 676.60 \\
Gas load curtailment cost & 0 & 0 \\
Heat load curtailment cost & 230.73 & 230.73 \\
\hline
\end{tabular}

\subsection{Comparison of the proposed method with the decentralized method based on regulated energy trading prices}

In this part, we compare the proposed method with the decentralized post-contingency dispatch method based on regulated energy trading prices, to analyze the effects of the proposed emergency energy market in stimulating the coordination of different participants. The regulated prices mean that the participants of IES jointly set the energy prices before the occurrence of contingencies. And when the IES encounters contingency events, these participants will trade energy with each other based on the pre-defined prices. It is a simple and common method to coordinate different participants of IES, which is similar to the constant bilateral price mechanism used in electricity markets [14]. The following three cases are defined:

Case 3: The electricity supply from the upper grid is interrupted from 3:00 to 10:00. To deal with this contingency, the proposed method based on distributed emergency energy trading is employed.

Case 4: The contingency is the same as Case 3. The emergency energy prices of EB, GB and GE are respectively fixed at $274.43 \$ / \mathrm{MWh}, 37.52 \$ / \mathrm{kcf}$ and $37.34 \$ / \mathrm{kcf}$, which are the average values of energy trading prices in 24 hours when the upper grid is in the outage state.

Case 5: The contingency is the same as Case 3. The emergency energy prices of EB, GB and GE are respectively fixed at 119.58 \$/MWh, $37.38 \$ / \mathrm{kcf}$ and $36.21 \$ / \mathrm{kcf}$, which are the average values of energy trading prices in 24 hours when the upper grid is in the normal condition.

Table 3 presents the post-contingency dispatch results of three cases. As shown in Table 3, compared to the decentralized method based on regulated prices (Case 4 and Case 5), the total operation cost and the load curtailment cost of IES are reduced with the proposed method (Case 3). It means the proposed dispatch method effectively enhances the 
economy and reliability of the entire IES. Moreover, based on the distributed energy trading mechanism, each participant can choose its favorable emergency energy trading prices and volumes with other participants, thereby maximizing its own profits. Therefore, compared to Case 4 and Case 5, the operation cost of ESO, GSO and HSO are simultaneously reduced in Case 3, meaning the proposed method is more beneficial to the participants of the IES.

Table 3. The post-contingency dispatch results of three cases

\begin{tabular}{lccc}
\hline \multicolumn{1}{c}{ Cost $(\$)$} & Case 3 & Case 4 & Case 5 \\
\hline Total operation cost of IES & 14571.33 & 14952.64 & 15007.64 \\
Total load curtailment cost & 472.19 & 1079.64 & 1234.75 \\
Operation cost of ESO & 907.04 & 965.82 & 1046.47 \\
Operation cost of GSO & 11498.23 & 11506.18 & 11513.43 \\
Operation cost of HSO & 2166.06 & 2480.64 & 2447.74 \\
\hline
\end{tabular}

To further analyze why the proposed method has a better performance in stimulating the coordination of participants, we take the energy trading at EB as an example. Fig. 10 and Fig. 11 present the emergency energy prices and trading volumes at EB. The results demonstrate that the regulated energy prices in Case 4 are too high, leading to the HSO (buyer) reduce the electricity demand of EB. On the contrary, the regulated prices in Case 5 are too low, which results in the ESO (seller) is unwilling to fully supply electricity to HSO. In the two cases, since the participants of IES are not fully motivated, the emergency energy trading volumes among participants are very limited. In practice, it is difficult (even impossible) to use fixed energy prices to achieve the optimal emergency energy allocation among participants, because the operational parameters (renewable energy output, consumer loads, etc.) of IES are changeable in the duration of contingencies. However, based on the proposed emergency energy market framework, the EETP can adjust the emergency energy prices in real-time to fully stimulate the energy trading among participants, thereby effectively optimizing the emergency energy allocation among different participants of the IES.

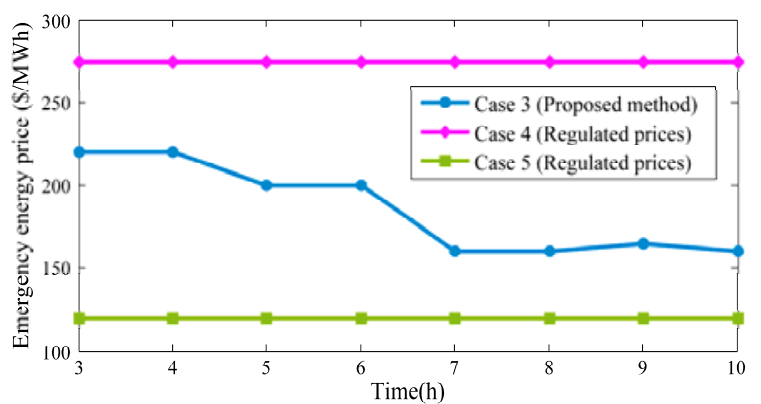

Fig. 10 The emergency energy prices at EB

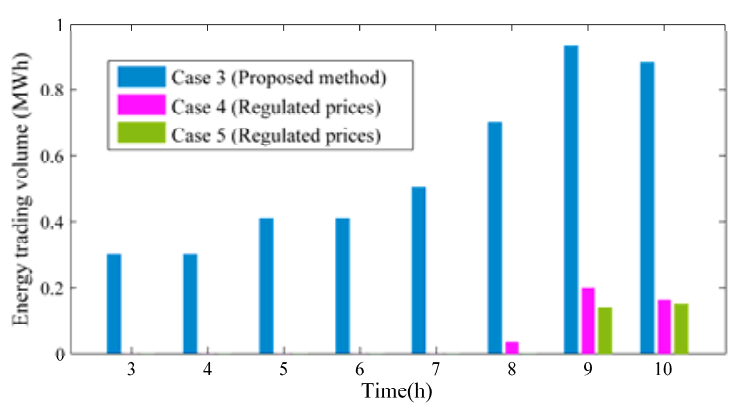

Fig. 11 The emergency energy trading volumes at EB

\subsection{The validation of the accuracy and computational efficiency of the proposed model and algorithm}

In this part, we firstly compare the proposed model with other energy network models used in the existing studies to 
validate the effectiveness of the linearization techniques used in this paper. Afterward, the proposed distributed emergency energy trading algorithm is compared with other distributed optimization algorithms.

\subsubsection{Effectiveness of the proposed energy network model}

The proposed energy network model is compared with other energy network models to validate the accuracy and computational efficiency of the linearization techniques used in this paper. The nonlinear electricity network (NLEN) model (2)-(23) is solved using the interior point method, and the optimization solver IPOPT is used because the solver Gurobi is not applicable for solving the NLEN model [5]. Moreover, the piecewise linearization techniques in [6, 44] are used to linearize the nonlinear gas network model and nonlinear heating network model respectively, which can convert a nonlinear optimization model to a mixed-integer linear programming (MILP) model. For each gas pipeline or heating pipeline, 20 segments are used. These two models are named the MILP-G model and MILP-H model, respectively. The contingency event that the electricity supply from the upper grid is interrupted from 8:00 to 16:00 is analyzed, and the post-contingency dispatch results using different energy network models are presented in Table 4.

Table 4. The post-contingency dispatch results using different energy network models

\begin{tabular}{ccccc}
\hline Model & Proposed model & NLEN model & MILP-G model & MILP-H model \\
\hline Total operation cost of IES (\$) & 18213.89 & 18335.98 & 18213.89 & 18213.89 \\
Number of iterations & 77 & 62 & 63 & 98 \\
Computation time $(\mathrm{sec})$ & 86.95 & 381.77 & 130.99 & 2461.64 \\
\hline
\end{tabular}

As shown in Table 4, the optimal objective results (total operation cost of IES) using different energy network models are very close. The relative error between the results of the proposed model and the NLEN model is $0.67 \%$, and the result of the proposed model is almost identical to the results of the MILP-G model and MILP-H model. It validates the accuracy of the linearization techniques used in this paper. Moreover, compared to other energy network models, the computational speed of the proposed model is faster. It takes $381.77 \mathrm{sec}$ to solve the NLEN model using the interior point method, which is more than 4 times compared to the computation time of the proposed model. Besides, it also takes more time to solve the MILP-G and MILP-H model. Especially for the MILP-H model, since there are 32 supply pipelines and 32 return pipelines in the heating system, the piecewise linearization method introduces a large number of binary variables to approximate the nonlinear heating network model, which brings a huge computational burden. In the postcontingency dispatch, the proposed model can effectively reduce the time spent in the decision-making process of participants.

\subsubsection{Effectiveness of the proposed distributed emergency energy trading algorithm}

The proposed distributed emergency energy trading algorithm is compared to the existing distributed optimization algorithms including the ADMM with constant penalty parameters (ADMM-c) in [37] and the auxiliary problem 
principle (APP) method in [45]. To easily compare the performance of algorithms, the initial values of coupling variables, emergency energy prices and penalty parameters are the same in these algorithms, and the maximum number of iterations is set to 200. Moreover, the results of the centralized post-contingency dispatch method are used as the benchmark. Considering the uncertainty of contingencies, multiple contingency events are set, as shown in Table 5. The postcontingency dispatch results using different optimization algorithms are presented in Table 6. In Table 6, the Obj means the optimal objective result (i.e., the total operation cost of IES during the given contingency).

Table 5. Contingency events of the test IES

\begin{tabular}{|c|c|c|c|c|c|}
\hline Contingency event & Event 1 & Event 2 & Event 3 & Event 4 & Event 5 \\
\hline Faulty component & $\begin{array}{l}\text { The electric line } \\
\text { from bus } 5 \text { to } 6\end{array}$ & Gas source GS1 & $\begin{array}{c}\text { The gas pipe from } \\
\text { node } 11 \text { to } 17\end{array}$ & Coal-fired boiler & $\begin{array}{l}\text { Heat supply pipe } \\
\text { from node } 5 \text { to } 11\end{array}$ \\
\hline Duration of contingency & $3: 00-9: 00$ & $1: 00-10: 00$ & $10: 00-20: 00$ & $12: 00-20: 00$ & $1: 00-8: 00$ \\
\hline
\end{tabular}

Table 6. The post-contingency dispatch results using different optimization algorithms

\begin{tabular}{cccccccccccc}
\hline & \multicolumn{2}{c}{ Centralized method } & \multicolumn{2}{c}{ Proposed algorithm } & \multicolumn{2}{c}{ ADMM-c algorithm } & \multicolumn{2}{c}{ APP algorithm } \\
\cline { 2 - 11 } & Obj $(\$)$ & $\begin{array}{c}\text { Time } \\
(\mathrm{sec})\end{array}$ & Obj $(\$)$ & Iterations & $\begin{array}{c}\text { Time } \\
(\mathrm{sec})\end{array}$ & Obj $(\$)$ & Iterations & $\begin{array}{c}\text { Time } \\
(\mathrm{sec})\end{array}$ & Obj (\$) & Iterations & $\begin{array}{c}\text { Time } \\
(\mathrm{sec})\end{array}$ \\
\hline 1 & 13099.37 & 3.41 & 13099.37 & 25 & 24.88 & - & - & - & 13099.37 & 149 & 147.29 \\
2 & 20038.76 & 4.45 & 20038.76 & 21 & 24.44 & 20038.76 & 71 & 81.44 & 20038.76 & 37 & 43.84 \\
3 & 27590.27 & 4.83 & 27590.27 & 21 & 26.46 & - & - & - & - & - \\
4 & 20089.45 & 4.54 & 20089.45 & 15 & 17.02 & 20089.45 & 94 & 109.29 & 20089.45 & 48 & 55.81 \\
5 & 13752.34 & 3.72 & 13752.34 & 20 & 19.59 & 13752.34 & 70 & 68.28 & 13752.34 & 36 & 35.31 \\
\hline
\end{tabular}

As shown in Table 6, for the 5 contingency events, the optimal objective results using the proposed distributed emergency energy trading algorithm are the same as the results using the centralized dispatch method, which indicates the accuracy of the proposed algorithm. However, for Event 1 and Event 3, the ADMM-c algorithm fails to achieve convergence within the maximum number of iterations. The APP algorithm also fails to achieve convergence for Event 3. Moreover, the number of iterations and computation time for the proposed algorithm are less than the ADMM-c and APP algorithm. It validates the proposed distributed emergency energy trading algorithm has higher computational efficiency in coordinating the post-contingency dispatch of different participants.

\subsection{The application on a larger scale integrated energy system}

The proposed post-contingency dispatch method is also applied to a larger scale IES. The IES includes a 61-bus electricity system modified from a real distribution system in Northwestern China, a modified 40-node natural gas system including two 20-node gas systems, and two 32-node heating systems. The maximal total load of electric consumers is 
19.4465 MW, the maximal total load of gas consumers is $100 \mathrm{kcf} / \mathrm{h}$, and the maximal total load of heat consumers is 4.328 MW. The topology of this IES is shown in Fig. 12, and the detailed data can be found in [46]. The tolerances of primal and dual residuals are set to 0.005 . Other data is the same as the small-scale IES. To validate the effectiveness of the proposed post-contingency dispatch method, three cases are defined:

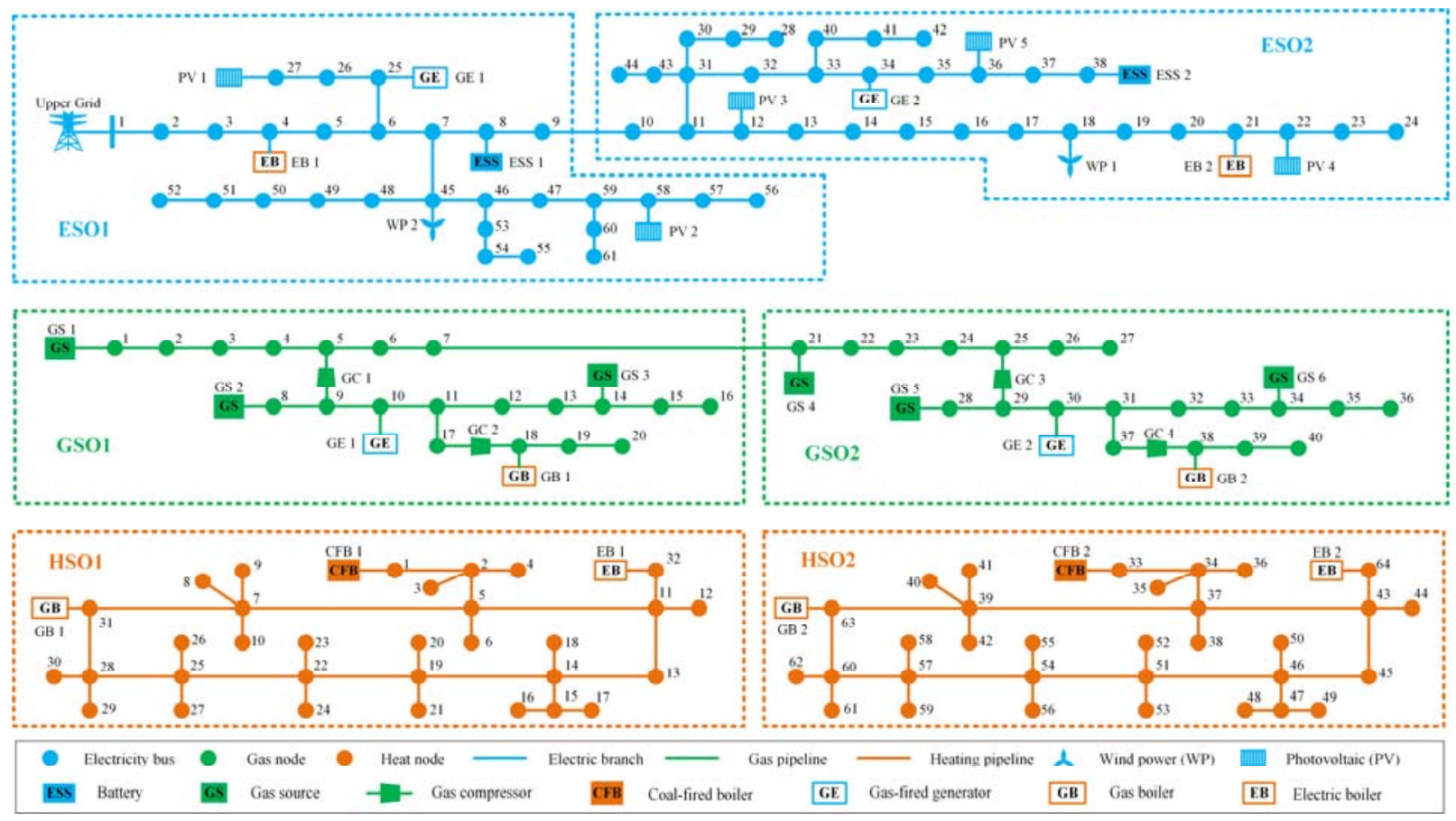

Fig. 12 The topology of a larger scale integrated energy system

Case 6: The electricity supply from the upper grid is interrupted from 1:00 to 12:00. The entire IES is managed by a centralized operator. Therefore, the centralized post-contingency dispatch method is used. The results are used as the benchmark.

Case 7: The contingency is the same as Case 6. The electricity, gas and heating systems of the IES are managed by one ESO, GSO and HSO, respectively. The proposed post-contingency dispatch method is employed.

Case 8: The contingency is the same as Case 6. However, the electricity system is respectively managed by ESO1 and ESO2, the gas system is respectively managed by GSO1 and GSO2, and two heating systems are managed by HSO1 and HSO2 individually, as shown in Fig. 12. This case represents a practical condition that there are multiple participants in the same energy field. And the proposed post-contingency dispatch method is also used to coordinate different participants. It is noteworthy that the coupling variables of the energy exchange on tie-lines and tie-pipes, the bus voltage on tie-lines, and the nodal gas pressure on tie-pipes also need to be considered in the post-contingency dispatch model, one can refer to [38] and [11].

Table 7 presents the post-contingency dispatch results for three cases. As shown in Table 7, the optimal objective 
results of three cases are close, which indicates the proposed method can effectively optimize the emergency energy dispatch of the IES when the scale of the system and the number of participants increase. Specifically, for Case 7, since the scale of the IES is large and the computational complexity of the dispatch model for each participant increases, it takes $173.49 \mathrm{sec}$ to coordinate the post-contingency dispatch of different participants. For Case 8, with the increase of the number of participants, the complexity of the dispatch model for each participant decreases, so the computation time for Case $8(114.89 \mathrm{sec})$ is less than Case 7. Overall, the proposed method can coordinate the post-contingency dispatch of the large-scale IES within minutes, which validates the computational efficiency of the proposed method in practice.

Table 7. The post-contingency dispatch results for Case 6-Case 8

\begin{tabular}{cccc}
\hline Case & Total operation cost of IES $(\$)$ & Total load curtailment cost $(\$)$ & Computation time (sec) \\
\hline Case 6 & 66912.46 & 13495.39 & 9.71 \\
Case 7 & 66911.07 & 13487.71 & 173.49 \\
Case 8 & 66907.79 & 13495.59 & 114.89 \\
\hline
\end{tabular}

In Case 8, since there are multiple participants in the same energy field (e.g., ESO1 and ESO2), the coordination of these participants also have considerable impacts on the post-contingency dispatch of the IES. Fig. 13 presents the emergency energy trading prices and power exchange between ESO1 and ESO2 during the contingency. The results show that, from 1:00 to 5:00, since the output of renewable energy is low, the electricity supply of the IES is in severe shortage, the emergency energy trading prices between ESO1 and ESO2 is high, and ESO2 purchases electricity from ESO1 to supply its important loads. On the contrary, from 7:00 to 12:00, with the increase of renewable energy power, the electricity shortage of ESO2 is in remission. Therefore, the emergency energy prices decreases and ESO2 sells electricity to ESO1. It indicates the proposed emergency energy market can also coordinate the post-contingency dispatch of different participants in the same energy field, thereby validating the effectiveness of the proposed method in practical conditions.

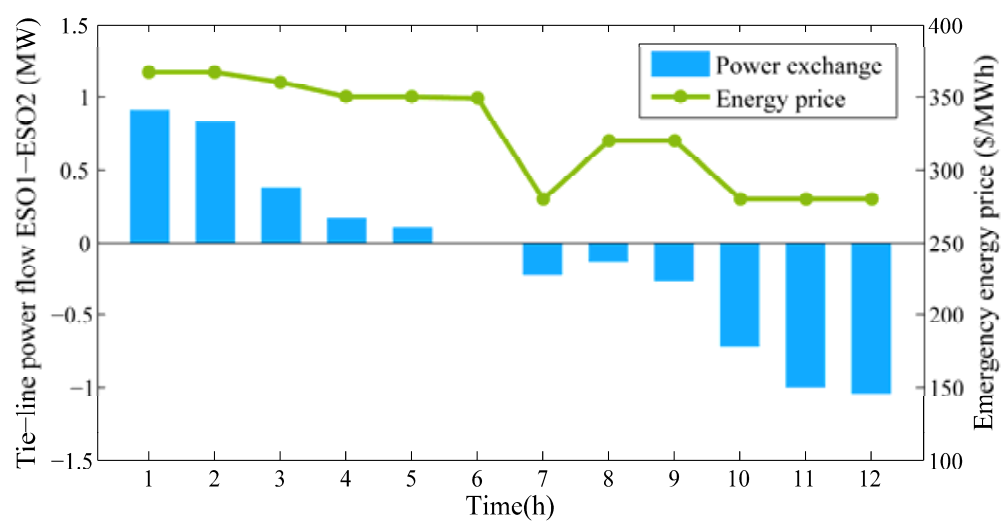

Fig. 13 The power exchange and emergency energy price between ESO1 and ESO2 


\section{Conclusions}

This paper presents a novel coordinated post-contingency dispatch method for the IES with multiple participants. An emergency energy market framework for IES is proposed to coordinate the energy allocation among participants based on the distributed energy trading mechanism. Moreover, considering the impacts of component failures, the postcontingency dispatch model of IES is formulated and several linearization techniques are employed to reduce the computational complexity. Furthermore, a distributed energy trading algorithm is developed to calculate emergency energy prices and coordinate different participants. The extensive case studies validate the proposed method can effectively coordinate the post-contingency dispatch of multiple independent participants, enhance the reliability and economy of the entire IES, as well as reducing the operation cost of participants during contingencies. The proposed method can also be used as a valuable supplement to current multi-energy markets, thereby reducing the risks of energy markets during contingencies.

\section{Acknowledgements}

This work was supported in part by the National Natural Science Foundation of China $(51637008,51977168)$, and in part by the National Key Research and Development Program of China (2016YFB0901900).

\section{References:}

[1] Good N, Ceseña E, Mancarella P. Ten questions concerning smart districts. Build Environ 2017; 118: 362-76.

[2] Massrur HR, Niknam T, Fotuhi-Firuzabad M, Nikoobakht A. Hourly electricity and heat demand response in the OEF of the integrated electricity-heat-natural gas system. IET Renew Power Gener 2019; 13(15): 2853-63.

[3] Liu F, Bie Z, Wang X. Day-ahead dispatch of integrated electricity and natural gas system considering reserve scheduling and renewable uncertainties. IEEE Trans Sustainable Energy 2019; 10(2): 646-58.

[4] Yao L, Wang X, Ding T, Wang Y, Wu X, Liu J. Stochastic day-ahead scheduling of integrated energy distribution network with identifying redundant gas network constraints. IEEE Trans Smart Grid 2019; 10(4), pp. 4309-22.

[5] Yu J, Guo L, Ma M, Kamel S, Li W, Song X. Risk assessment of integrated electrical, natural gas and district heating systems considering solar thermal CHP plants and electric boilers. Int J Electr Power Energy Syst 2018; 103: 277-87.

[6] Zeng Z, Ding T, Xu Y, Yang Y, Dong Z. Reliability evaluation for integrated power-gas systems with power-to-gas and gas storages. IEEE Trans Power Syst 2020; 35(1): 571-83.

[7] Guo Z, Li G, Zhou M, Feng W. Resilient configuration approach of integrated community energy system considering integrated demand response under uncertainty. IEEE Access 2019; 7: 87513-33.

[8] Chen Y, Wei W, Liu F, Sauma EE, Mei S. Energy trading and market equilibrium in integrated heat-power distribution systems. IEEE Trans Smart Grid 2019; 10(4): 4080-94.

[9] Wen Y, Qu X, Li W, Liu X, Ye X. Synergistic operation of electricity and natural gas networks via ADMM. IEEE Trans Smart Grid 2018; 9(5): 4555-65.

[10] Wang C, Yan C, Li G, Liu S, Bie Z. Risk assessment of integrated electricity and heat system with independent energy operators based on Stackelberg game. Energy 2020; 198. 117349. 
[11] Kim H, Lee J, Bahrami S, Wong VWS. Direct energy trading of microgrids in distribution energy market. IEEE Trans Power Syst 2020; 35(1): 639-51.

[12] Morstyn T, McCulloch MD. Multiclass energy management for peer-to-peer energy trading driven by prosumer preferences. IEEE Trans Power Syst 2019; 34(5): 4005-14.

[13] Nunna HSVSK., Srinivasan D. Multiagent-based transactive energy framework for distribution systems with smart Microgrids. IEEE Trans Ind Inf 2017; 13(5): 2241-50.

[14] Farzin H, Ghorani R, Fotuhi-Firuzabad M, Moeini-Aghtaie M. A market mechanism to quantify emergency energy transactions value in a multi-microgrid system. IEEE Trans Sustainable Energy 2019; 10(1): 426-37.

[15] Sorknæs P, Lund H, Skov IR, Djørup S, Skytte K, Morthorst PE, et al. Smart Energy Markets-Future electricity, gas and heating markets. Renew Sustain Energy Rev 2020; 119. 109655.

[16] Stiphout AV, Virag A, Kessels K, Deconinck G. Benefits of a multi-energy day-ahead market. Energy 2018; 165: 651-61.

[17] Luo Y, Zhang X, Yang D, Sun Q, Zhang H. Optimal operation and cost-benefit allocation for multi-participant cooperation of integrated energy system. IET Gener Transm Distrib 2019; 13(22): 5239-47.

[18] Wang C, Wei W, Wang J, Wu L, Liang Y. Equilibrium of interdependent gas and electricity markets with marginal price based bilateral energy trading. IEEE Trans Power Syst 2018; 33(5): 4854-67.

[19] Yang H, Zhang Y, Ma Y, Zhang D, Sun L, Xia S. Reliability assessment of integrated energy system considering the uncertainty of natural gas pipeline network system. IET Gener Transm Distrib 2019; 13(22): 5033-41.

[20] Li R, Wei W, Mei S, Hu Q, Wu Q. Participation of an energy hub in electricity and heat distribution markets: an MPEC approach. IEEE Trans Smart Grid 2019; 10(4): 3641-53.

[21] Huang M, Wei Z, Ju P, Wang J, Chen S. Incentive-compatible market clearing for a two-stage integrated electricity-gas-heat market. IEEE Access 2019; 7: 120984-96.

[22] Li G, Bie Z, Kou Y, Jiang J, Bettinelli M. Reliability evaluation of integrated energy systems based on smart agent communication. Appl Energy 2016; 167: 397-406.

[23] Han S, Han S, Aki H. A practical battery wear model for electric vehicle charging applications. Appl Energy 2014; 113: 1100-08.

[24] Pazouki S, Mohsenzadeh A, Ardalan S, Haghifam MR. Optimal place, size, and operation of combined heat and power in multi carrier energy networks considering network reliability, power loss, and voltage profile. IET Gener Transm Distrib 2016; 10(7): $1615-21$.

[25] Ding T, Liu S, Wu Z, Bie Z. Sensitivity-based relaxation and decomposition method to dynamic reactive power optimisation considering DGs in active distribution networks. IET Gener Transm Distrib 2017; 11(1): 37-48.

[26] Safdarian A, Fotuhi-Firuzabad M, Aminifar F, Lehtonen M. A new formulation for power system reliability assessment with AC constraints. Int J Electr Power Energy Syst 2014; 56: 298-306.

[27] Ben-Tal A, Nemirovski A. On polyhedral approximations of the second-order cone. Math Oper Res 2001; 26(2): 193-205.

[28] Chen S, Wei Z, Sun G, Wang D, Zang H. Steady state and transient simulation for electricity-gas integrated energy systems by using convex optimisation. IET Gener Transm Distrib 2018; 12(9): 2199-2206.

[29] Ordoudis C, Pinson P, Morales JM. An integrated market for electricity and natural gas systems with stochastic power producers. Eur J Oper Res 2019; 272: 642-54.

[30] He C, Zhang X, Liu T, Wu L. Distributionally robust scheduling of integrated gas-electricity systems with demand response. IEEE Trans Power Syst 2019; 34(5): 3791-3803.

[31] Fodstad M, Midthun KT, Tomasgard A. Adding flexibility in a natural gas transportation network using interruptible transportation services. Eur J Oper Res 2015; 243: 647-57.

[32] Liu X, Wu J, Jenkins N, Bagdanavicius A. Combined analysis of electricity and heat networks. Appl Energy 2016; 162: 1238-50.

[33] Wang P. Reliability of district heating system based on graph theory. PhD Thesis. Harbin Institute of Technology; 2010.

[34] Wang P, Zou P. Simulation of distributed pump systems under failure conditions. Proceedings of Second International Conference on Modeling and Simulation Vol.3, Manchester, England, 2009; pp. 118-123.

[35] Pirouti M, Bagdanavicius A, Ekanayake J, Wu J, Jenkins N. Energy consumption and economic analyses of a district heating 
network. Energy 2013; 57: 149-59.

[36] Castro PM. Tightening piecewise McCormick relaxations for bilinear problems. Comput Chem Eng 2015; 72: 300-11.

[37] Boyd S, Parikh N, Chu E, Peleato B, Eckstein J. Distributed optimization and statistical learning via the alternating direction method of multipliers. Foundations and Trends in Machine Learning 2010; 3(1): 1-122.

[38] Wu G, Xiang Y, Liu J, Gou J, Shen X, Huang Y, et al. Decentralized day-ahead scheduling of multi-area integrated electricity and natural gas systems considering reserve optimization. Energy 2020; 198. 117271.

[39] Zhang T, Zhang W, Zhao Q, Du Y, Chen J, Zhao J. Distributed Real-Time State Estimation for Combined Heat and Power Systems. J Mod Power Syst Clean Energy 2020, early access, doi: 10.35833/MPCE.2020.000052.

[40] Yang H, Li M, Jiang Z, Zhang P. Multi-time scale optimal scheduling of regional integrated energy systems considering integrated demand response. IEEE Access 2020; 8: 5080-90.

[41] Baran ME, Wu FF. Network reconfiguration in distribution systems for loss reduction and load balancing. IEEE Trans Power Deliv 1989; 4(2): 1401-7.

[42] He C, Dai C, Wu L, Liu T. Robust network hardening strategy for enhancing resilience of integrated electricity and natural gas distribution systems against natural disasters. IEEE Trans Power Syst 2018; 33(5): 5787-98.

[dataset] [43] Wang C. 'Data_IEEE33NGS20DHS32.xlsx', GitHub Data, 2020. https://github.com/CanboyEE/Data_IES_case.

[44] Wang X, Bie Z, Liu F, Kou Y, Jiang L. Bi-level planning for integrated electricity and natural gas systems with wind power and natural gas storage. Int J Electr Power Energy Syst 2020; 118. 105738.

[45] Liu JH, Chu CC. Iterative Distributed Algorithms for Real-Time Available Transfer Capability Assessment of Multiarea Power Systems. IEEE Trans Smart Grid 2015; 6(5): 2569-78.

[dataset] [46] Wang C. 'Data_Real61NGS40NHS64_IES.xlsx', GitHub Data, 2020. https://github.com/CanboyEE/Data_IES_case. 